TPI-MINN-99/14

NUC-MINN-99/6-T

UMN-TH-1750

March 1999

\title{
Three Lectures on the Physics of Small $x$ and High Gluon Density ${ }^{1}$
}

\author{
Larry McLerran
}

Theoretical Physics Institute, University of Minnesota, Minneapolis, MN 55455

Abstract: In these lectures, I shall discuss small x physics and the consequences of the high gluon density which arises as x decreases. I argue that an understanding of this problem would lead to knowledge of the high energy asymptotics of hadronic processes. The high gluon density should allow a first principles computation of these asymptotics from QCD.

\section{LECTURE I: LOTS OF PROBLEMS}

\section{A Introduction}

I think we all believe that QCD describes hadronic physics. It has been tested in a variety of environments. For high energy short distance phenomena, perturbative QCD computations successfully confront experiment. In lattice Monte-Carlo computations, one gets a successful semi-quantitative description of hadronic spectra, and perhaps in the not too distant future one will obtain precise quantitative agreement.

At present, however, all analytic computations and all precise QCD tests are limited to the small class of problems which correspond to short distance physics. Here there is some characteristic energy transfer scale $E$, and one uses asymptotic freedom,

$$
\alpha_{S}(E) \rightarrow 0
$$

as $E \rightarrow \infty$

One question which we might ask is whether there are any non-perturbative "simple phenomena" which arise from QCD which are worthy of further effort. The questions I would ask before I would become interested in understanding such phenomena are

1) Lectures given at the VIII'th Mexican School of Particles and Fields 
- Is the phenomenon simple and pervasive?

- Is it reasonably plausible that one can understand the phenomena from first principles, and compute how it would appear in nature?

I will in this lecture try to explain a wide class of phenomena in QCD which are pervasive, and appear to follow simple patterns. I will then try to explain why I believe that these phenomena can be simply understood within QCD.

\section{B Total Cross Sections at Asymptotic Energy}

Computing total cross section as $E \rightarrow \infty$ is one of the great unsolved problems of QCD. Unlike for processes which are computed in perturbation theory, it is not required that any energy transfer become large as the total collision energy $E \rightarrow \infty$. Computing a total cross section for hadronic scattering therefore appears to be intrinsically non-perturbative. In the 60's and early 70's, Regge theory was extensively developed in an attempt to understand the total cross section. The results of this analysis were to my mind inconclusive, and certainly can not be claimed to be a first principles understanding from QCD.

The total cross section for $\bar{p} p$ collisions is shown in Fig. 1. Typically, it is assumed that the total cross section grows as $\ln ^{2} E$ as $E \rightarrow \infty$. This is the so called Froisart bound which corresponds to the maximal growth allowed by unitarity of the $\mathrm{S}$ matrix. Is this correct? Is the coefficient of $\ln ^{2} E$ universal for all hadronic precesses? Why is the unitarity limit saturated? Can we understand the total cross section from first principles in QCD? Is it understandable in weakly coupled QCD, or is it an intrinsically non-perturbative phenomenon?

\section{How Are Particle Produced in High Energy Collisions?}

In Fig. 2, I plot the multiplicity of produced particles in $p p$ and in $\bar{p} p$ collisions. The last three open circles correspond to the $\bar{p} p$ collisions with the multiplicity at zero energy subtracted. The remaining open circles to $p p$. The x's are $\bar{p} p$ collisions without the multiplicity at zero energy subtracted. Notice that the open circles fall on roughly the same curve. The implication is that whatever is causing the increase in multiplicity in these collisions may be from the same mechanism.

The obvious question is can we compute $N(E)$, the total multiplicity of produced particles as a function of energy?

At this point it is useful to develop some mathematical tools. I will introduce some useful kinematic variables: light cone coordinates. Let the light cone longitudinal momenta be

$$
p^{ \pm}=\frac{1}{\sqrt{2}}\left(E \pm p_{z}\right)
$$

Note that the invariant dot product 


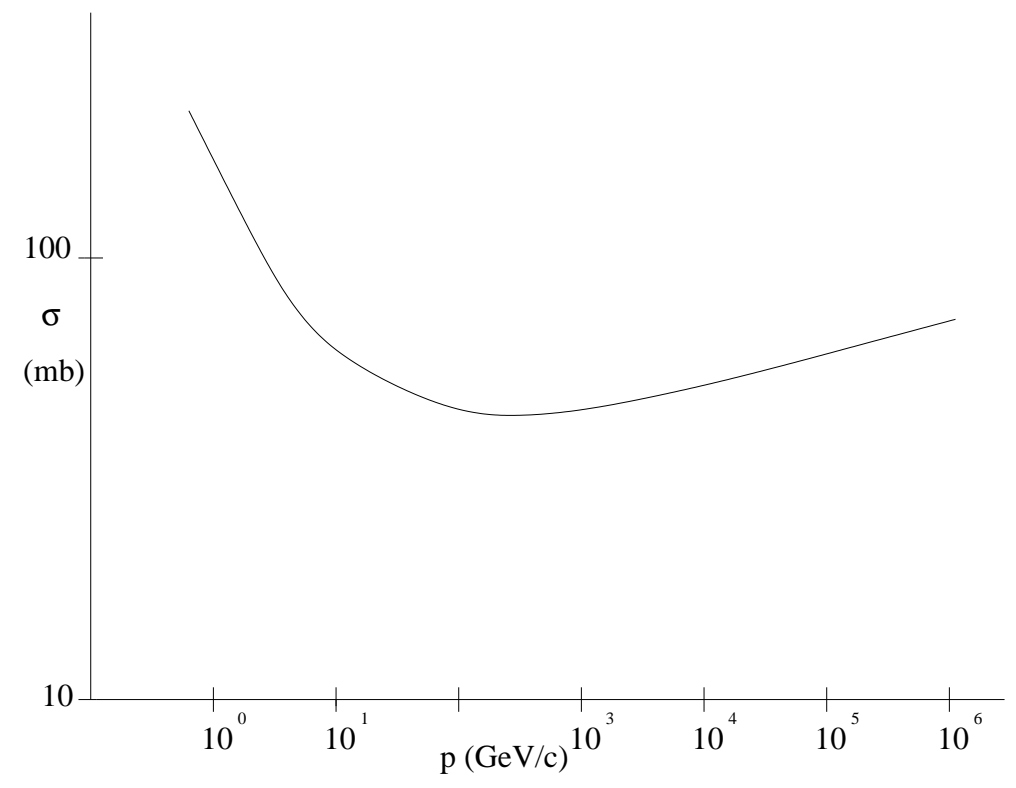

FIGURE 1. The total cross section for $p \bar{p}$ collisions

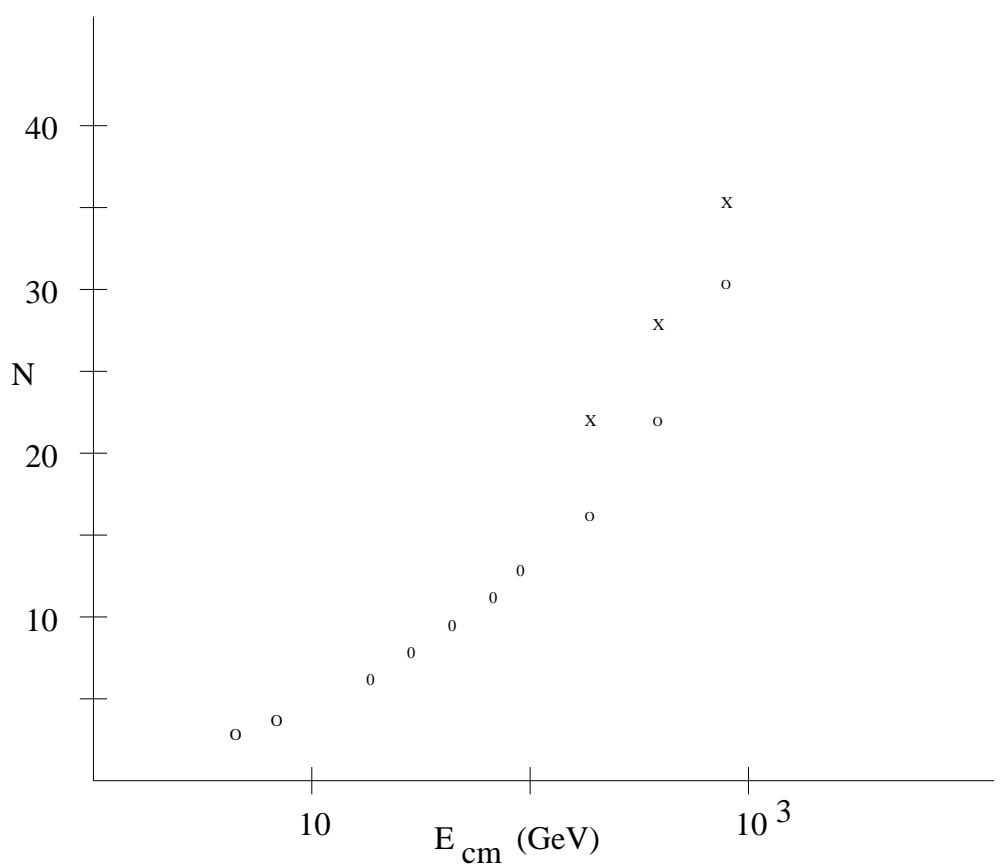

FIGURE 2. Multiplicity of produced particles in $p p$ and $p \bar{p}$ collisions 


$$
p \cdot q=p_{t} \cdot q_{t}-p^{+} q^{-}-p^{-} q^{+}
$$

and that

$$
p^{+} p^{-}=\frac{1}{2}\left(E^{2}-p_{z}^{2}\right)=\frac{1}{2}\left(p_{T}^{2}+m^{2}\right)=\frac{1}{2} m_{T}^{2}
$$

This equation defines the transverse mass $m_{T}$. (Please note that my metric is the negative of that conventionally used in particle physics. An unfortunate consequence of my education. Students, please feel free to convert everything to your favorite metric.)

Consider a collision in the center of mass frame as shown in Fig. 3. The right moving particle has $p_{1}^{+} \sim \sqrt{2}\left|p_{z}\right|$ and $p_{1}^{-} \sim \frac{1}{2 \sqrt{2}} m_{T}^{2} /\left|p_{z}\right|$. For the colliding

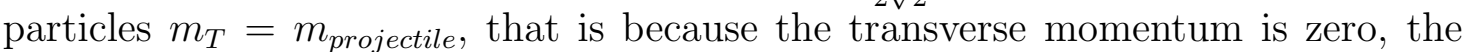
transverse mass equals the particle mass For particle 2 , we have $p_{2}^{+}=p_{1}^{-}$and $p_{2}^{-}=p_{1}^{+}$.

If we define the Feynman $x$ of a produced pion as

$$
x=p_{\pi}^{+} / p_{1}^{+}
$$

then $0 \leq x \leq 1$. The rapidity of a pion is defined to be

$$
y=\frac{1}{2} \ln \left(p_{\pi}^{+} / p_{\pi}^{-}\right)=\frac{1}{2} \ln \left(2 p^{+2} / m_{T}^{2}\right)
$$

For pions, the transverse mass includes the transverse momentum of the pion.

The pion rapidity is always in the range $-y_{C M} \leq y \leq y_{C M}$ where $y_{C M}=$ $\ln \left(p^{+} / m_{\text {projectile }}\right)$ All the pions are produced in a distribution of rapidities within this range.

These definitions are useful, among other reasons, because of their simple properties under longitudinal Lorentz boosts: $p^{ \pm} \rightarrow \kappa^{ \pm 1} p^{ \pm}$where $\kappa$ is a constant. Under boosts, the rapidity just changes by a constant. (Students, please check this relationship for momenta under boosts.)

A typical distribution of pions is shown in Fig. 4. It is convenient in the center of mass frame to think of the positive rapidity pions as somehow related to the right moving particle and the negative rapidity particles as related to the left moving particles. We define $x=p^{+} / p_{\text {projectile }}^{+}$and $x^{\prime}=p^{-} / p_{\text {projectile }}^{-}$and use $x$ for positive rapidity pions and $x^{\prime}$ for negative rapidity pions.

Of course more than just pions are produced in high energy collisions. The variables we just presented easily generalize to these particles.

Several theoretical issues arise in multiparticle production. Can we compute $d N / d y$ ? or even $d N / d y$ at $y=0$ ? How does the average transverse momentum of produced particles $\left\langle p_{T}\right\rangle$ behave with energy? What is the ratio of produced strange/nonstrange, and corresponding rations of charm, top, bottom etc at $y=0$ as the center of mass energy approaches infinity?

Does multiparticle production as $E \rightarrow \infty$ at $y=0$ become 


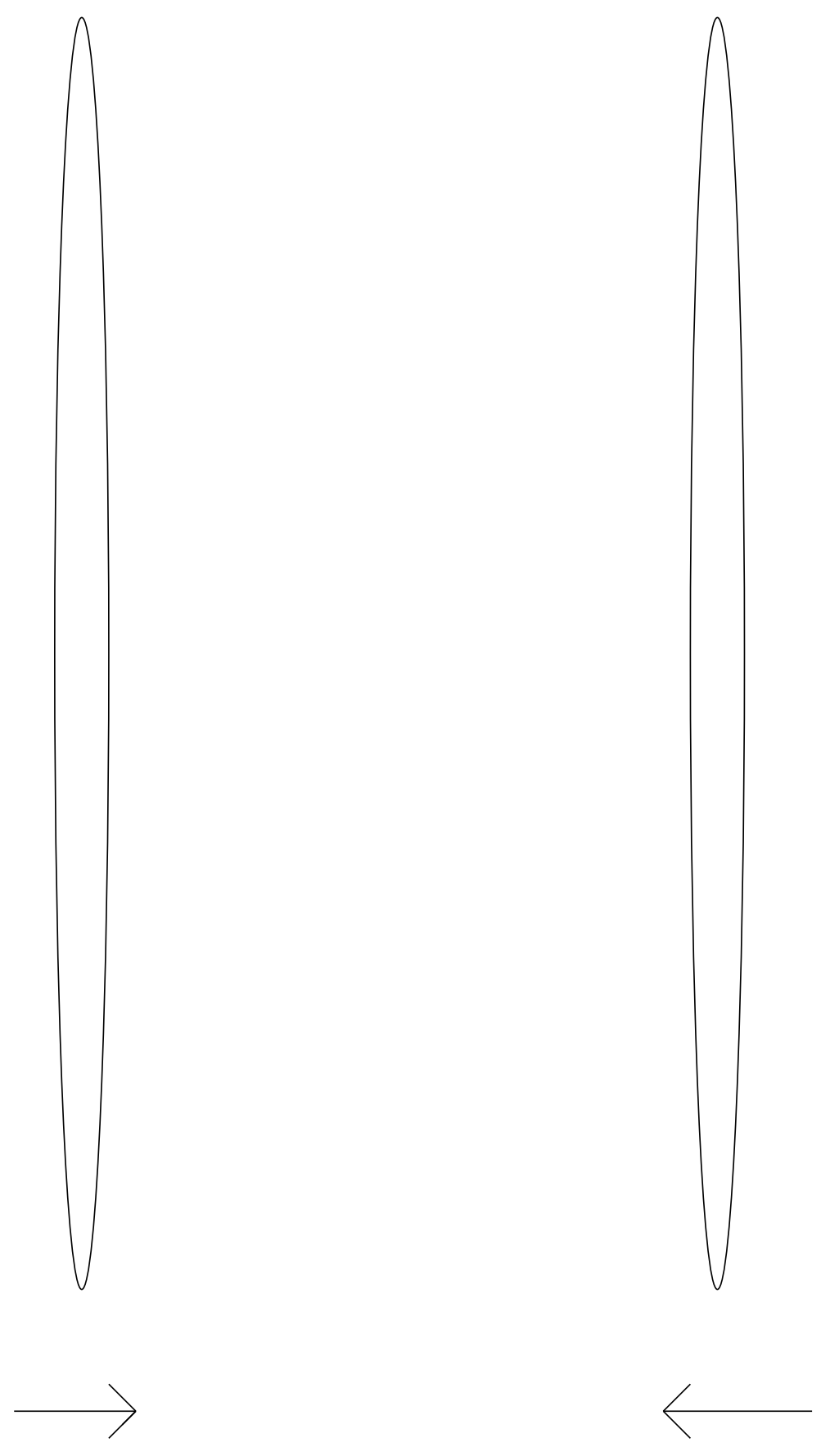

FIGURE 3. The collisions of two ultrarelativistic nuclei as seen in the center of mass frame 


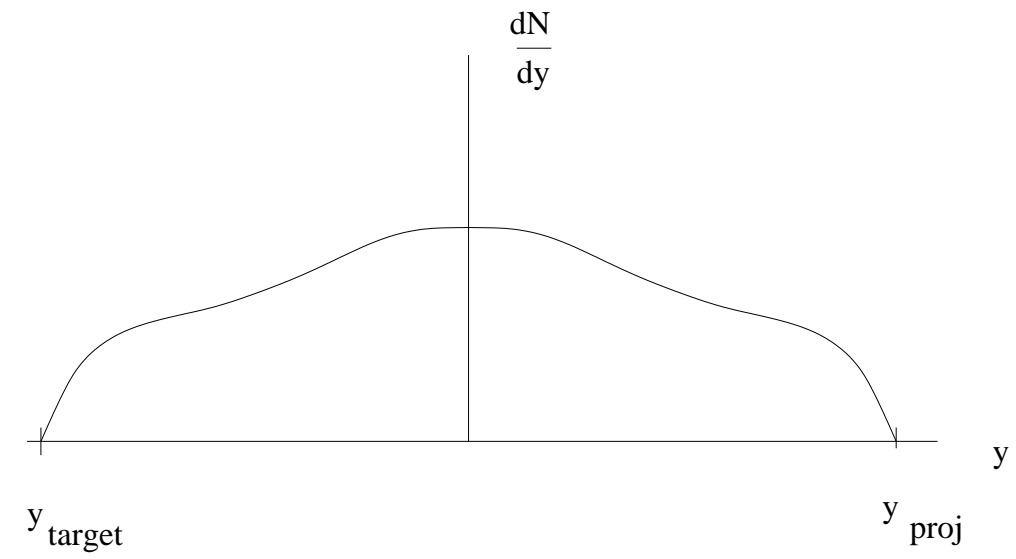

FIGURE 4. A typical pion rapidity distributions for hadronic collisions.

- Simple?

- Understandable?

- Computable?

\section{Deep Inelastic Scattering}

In Fig. 5, a cartoon of deep inelastic scattering is shown. Here an electron emits a virtual photon which scatters from a quark in a hadron. The momentum and energy transfer of the electron is measured, and the results of the break up are not. In these lectures, we cannot develop the theory of deep inelastic scattering. Suffice it to say, that this measurement is sufficient at large momenta transfer $Q^{2}$ to measure the distributions of quarks in a hadron.

To describe the quark distributions, it is convenient to work in a reference frame where the hadron has a large longitudinal momentum $p_{\text {hadron }}^{+}$. The corresponding light cone momentum of the constituent is $p_{\text {constituent. We define }}^{+}$ $x=p_{\text {constituent }}^{+} / p_{\text {hadron }}^{+}$. (This $\mathrm{x}$ variable is equal to the Bjorken $\mathrm{x}$ variable, which can be defined in a frame independent way. In this frame independent definition, $x=Q^{2} / 2 p \cdot Q$ where $p$ is the momentum of the hadronic target and $Q$ is the momentum of the virtual photon. Students, please check that this is true.) The cross section which one extracts in deep inelastic scattering can be related to the distributions of quarks inside a hadron, $d N / d x$.

It is useful to think about the distributions as a function of rapidity. We define this for deep inelastic scattering as

$$
y=y_{\text {hadron }}-\ln (1 / x)
$$

and the invariant rapidity distribution as 


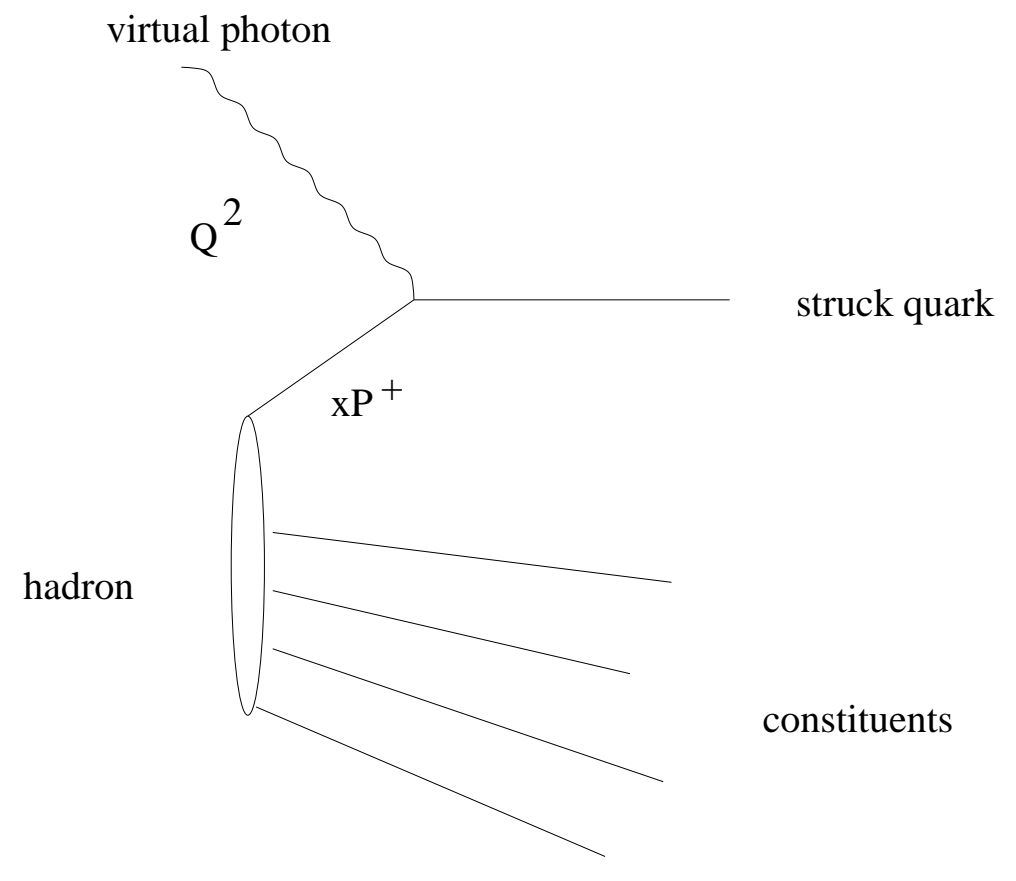

FIGURE 5. A cartoon of deep inelastic scattering.

$$
d N / d y=x d N / d x
$$

In Fig. 6, a typical $d N / d y$ distribution for a constituent gluons of a hadron is shown. This plot is similar to the rapidity distribution of produced particles in deep inelastic scattering. The main difference is that we have only half of the plot, corresponding to the left moving hadron in a collision in the center of mass frame.

We shall later argue that there is in fact a relationship between the structure functions as measured in deep inelastic scattering and the rapidity distributions for particle production. We will argue that the gluon distribution function is in fact proportional to the pion rapidity distribution.

The small $\mathrm{x}$ problem is that in experiments at Hera, the rapidity distribution function for quarks grows as the rapidity difference between the quark and the hadron grows. This growth appears to be more rapid than simply $\left|y_{\text {proj }}-y\right|$ or $\left(y_{\text {proj }}-y\right)^{2}$, and various theoretical models based on the original considerations of Lipatov and colleagues suggest it may grow as an exponential in $\left|y_{\text {proj }}-y\right|$. [1] If the rapidity distribution grew at most as $y^{2}$, then there would be no small $\mathrm{x}$ problem. We shall try to explain the reasons for this later in this lecture.

In Fig. 7, the Zeus data for the gluon structure function is shown. [2] I have plotted the structure function for $Q^{2}=7 \mathrm{GeV}^{2}$ and $20 \mathrm{GeV}^{2}$. The structure function depends upon the resolution of the probe, that is $Q^{2}$. Note the rise of $x g(x)$ at small $\mathrm{x}$, this is the small $\mathrm{x}$ problem. I have also plotted the total multiplicity of produced particles in $p p$ and $\bar{p} p$ collisions in the open circles on the same plot. Here I have used that $y=\log \left(E_{c m} / 1 G e V\right)$ for the pion production data. This is 


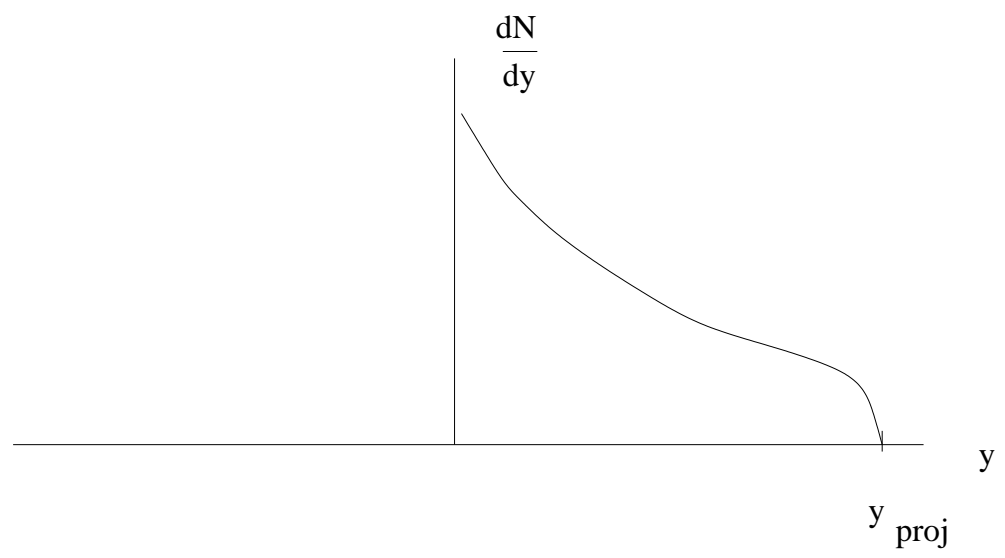

FIGURE 6. A rapidity distribution for gluons in the hadron wavefunction.

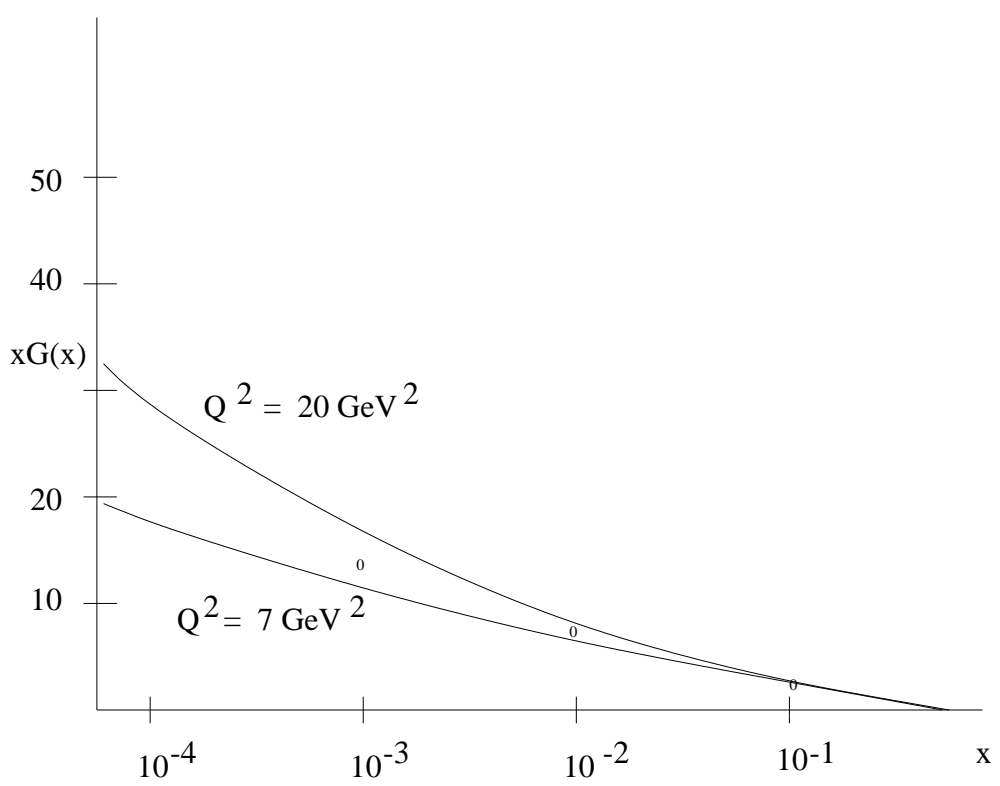

FIGURE 7. The Zeus data for the gluon structure functions. Error bars on the data are not shown, but are about 10 percent 


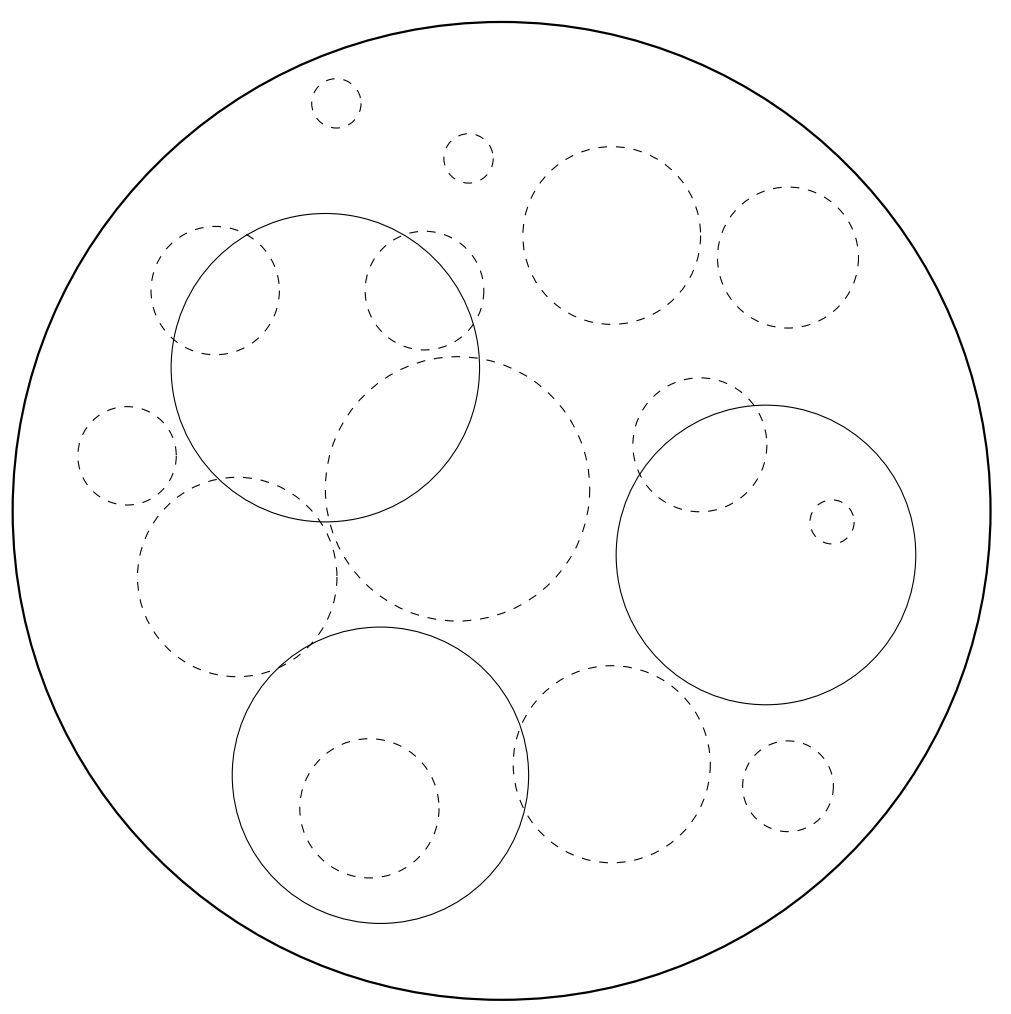

FIGURE 8. A picture of a hadron viewed head on.

approximately the maximal value of rapidity difference between centrally produced pions and the projectile rapidity. The total multiplicity has been rescaled so that at small $\mathrm{x}$, it matches the gluon structure functions. (Strictly speaking, we should have plotted the total multiplicity at $y=0$, but this is hard to extract from the data. If the distribution is an exponential in rapidity, then up to a constant these would be proportional.). Observe that the qualitative similarity between the gluon structure function and the total multiplicity.

Why is the small $\mathrm{x}$ rise in the gluon distribution a problem? Consider Fig. 8, where we view hadron head on. [3] The constituents are the valence quarks, shown as solid circles, and the gluons and sea quarks shown as circles with dashed lines. As we add more and more constituents, the hadron becomes more and more crowded. If we were to try to measure these constituents with say an elementary photon probe, as we do in deep inelastic scattering, we might expect that the hadron would become so crowded that we could not ignore the shadowing effects of constituents as we make the measurement. (Shadowing means that some of the partons are obscured by virtue of having another parton in front of them. For hard spheres, for example, this would result in a decrease of the scattering cross section relative to what is expected from incoherent independent scattering.)

In fact, in deep inelastic scattering, we are measuring the cross section for 


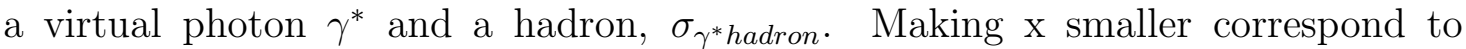
increasing the energy of the interaction (at fixed $Q^{2}$ ). An exponential growth in the rapidity corresponds to power law growth in $1 / x$, which in turn implies power law growth with energy. This growth, if it continues forever, violates unitarity. The Froissart bound will allow at most $\ln ^{2}(1 / x)$. (The Froissart bound is a limit on how rapidly a total cross section can rise. It follows from the unitarity of the scattering matrix.)

We shall later argue that in fact the distribution functions at fixed $Q^{2}$ do in fact saturate and cease growing so rapidly at high energy. The total number of gluons however demands a resolution scale, and we will see that the natural intrinsic scale is growing at smaller values of $\mathrm{x}$, so that effectively, the total number of gluons within this intrinsic scale is always increasing. The quantity

$$
\Lambda^{2}=\frac{1}{\pi R^{2}} \frac{d N}{d y}
$$

defines this intrinsic scale. Here $\pi R^{2}$ is the cross section for hadronic scattering from the hadron. For a nucleus, this is well defined. For a hadron, this is less certain, but certainly if the wavelngths of probes are small compared to $R$, this should be well defined. If

$$
\Lambda^{2}>>\Lambda_{Q C D}^{2}
$$

as the Hera data suggests, then we are dealing with weakly coupled QCD since $\alpha_{S}(\Lambda)<<1$.

Even though QCD may be weakly coupled at small $\mathrm{x}$, that does not mean the physics is perturbative. There are many examples of nonperturbative physics at weak coupling. An example is instantons in electroweak theory, which lead to the violation of baryon number. Another example is the atomic physics of highly charged nuclei. The electron propagates in the background of a strong nuclear Coulomb field, but on the other hand, the theory is weakly coupled and there is a systematic weak coupling expansion which allows for systematic computation of the properties of high $\mathrm{Z}$ ( $\mathrm{Z}$ is the charge of the nucleus) atoms.

If the theory is local in rapidity, then the only parameter which can determine the physics at that rapidity is $\Lambda^{2}$. (Locality in rapidity means that there are not long range correlations in the hadronic wavefunction as a function of rapidity. In pion production, it is known that except for overall global conserved quantities such as energy and total charge, such correlations are of short range.) Note that if only $\Lambda^{2}$ determines the physics, then in an approximately scale invariant theory such as QCD, a typical transverse momentum of a constituent will also be of order $\Lambda^{2}$. If $\Lambda^{2}>>1 / R^{2}$, where $R$ is the radius of the hadron, then the finite size of the hadron becomes irrelevant. Therefore at small enough $\mathrm{x}$, all hadrons become the same. The physics should only be controlled by $\Lambda^{2}$.

There should therefore be some equivalence between nuclei and say protons. When their $\Lambda^{2}$ values are the same, their physics should be the same. We can take an empirical parameterization of the gluon structure functions as 


\section{ZEUS 1995}

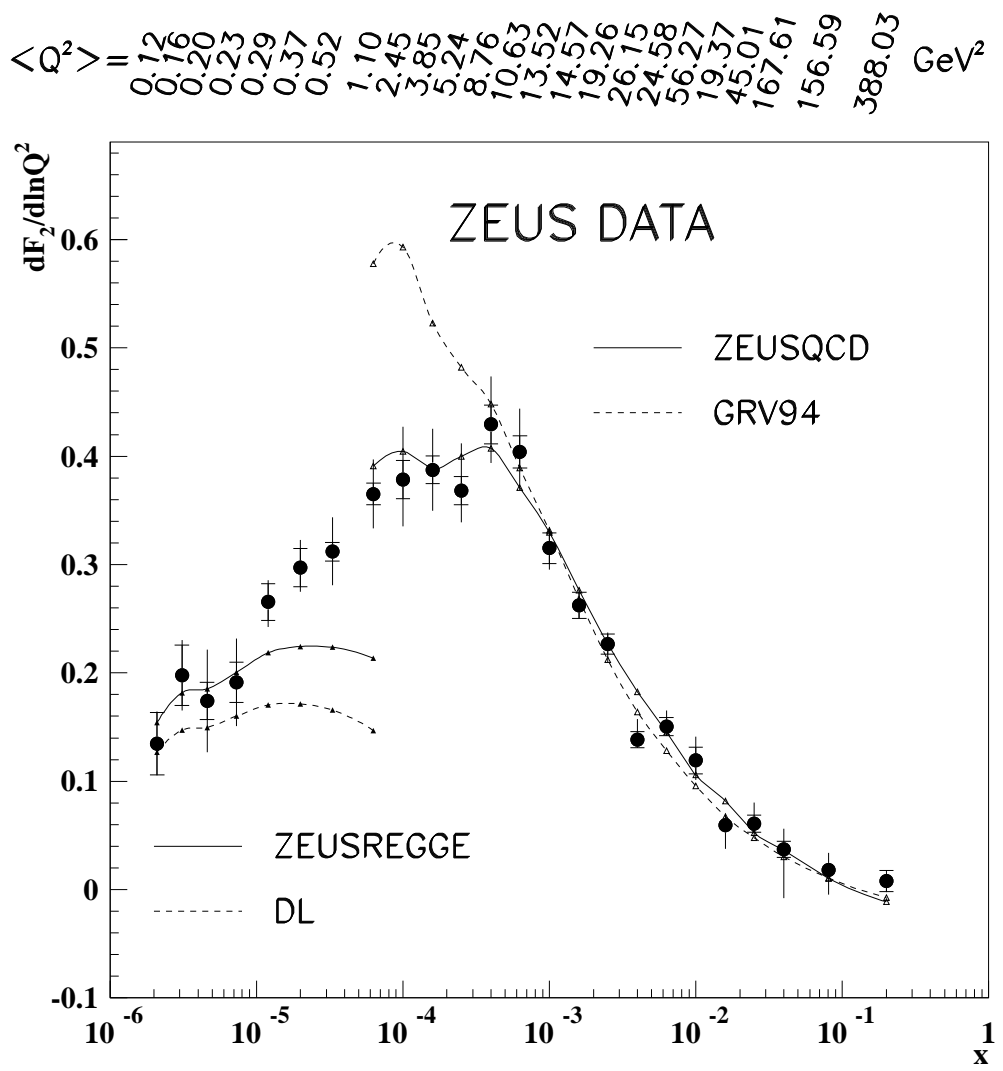

FIGURE 9. The Caldwell plot for the derivative of $F_{2}$

$$
\frac{1}{\pi R^{2}} \frac{d N}{d y} \sim \frac{A^{1 / 3}}{x^{\delta}}
$$

where $\delta \sim .2-.3$. This suggests that there should be the following correspondences:

- $\mathrm{RHIC}$ with nuclei $\sim$ Hera with protons

- LHC with nuclei Hera with nuclei

To get some rough idea of what the scales are which are important, consider the Caldwell plot of the Zeus data shown in Fig. 9. [2] The function $d F_{2} / d \ln Q^{2}$, the derivative of the structure function $F_{2}$ is a function of both $x$ and $Q^{2}$. The Caldwell plot takes a slice of this data in the $Q^{2}, x$ plane. The important thing to observe is the qualitative change in the behavior of the function at $Q^{2} \sim 3 \mathrm{GeV}^{2}$ at an $x \sim 10^{-4}$. Until we get to this value, the curve is adequately parameterized using DGLAP evolution equations and GRV parameterization of the gluon distribution functions. It is suggestive that the turnover has something to do with the physics of 
high gluon density. If so the typical scale associated with gluon momenta is rather large.

Since the physics of high gluon density is weak coupling we have the hope that we might be able to do a first principle calculation of

- the gluon distribution function

- the quark and heavy quark distribution functions

- the intrinsic $p_{T}$ distributions quarks and gluons

We can also suggest a simple escape from unitarity arguments which suggest that the gluon distribution function must not grow at arbitrarily small $\mathrm{x}$. The point is that at smaller $\mathrm{x}$, we have larger $\Lambda$ and correspondingly larger $p_{T}$. A typical parton added to the hadron has a size of order $1 / p_{T}$. Therefore although we are increasing the number of gluons, we do it by adding in more gluons of smaller and smaller size. A probes of size resolution $\Delta x \geq 1 / p_{T}$ at fixed $Q$ will not see partons smaller than this resolution size. They therefore do not contribute to the fixed $Q^{2}$ cross section, and there is no contradiction with unitarity.

\section{E Heavy Ion Collisions}

In Fig. 10, the standard lightcone cartoon of heavy ion collisions is shown. [4] To understand the figure, imagine we have two Lorentz contracted nuclei approaching one another at the speed of light. Since they are well localized, they can be thought of as sitting at $x^{ \pm}=0$, that is along the light cone, for $t<0$ At $x^{ \pm}=0$, the nuclei collide. To analyze this problem for $t \geq 0$, it is convenient to introduce a time variable which is Lorentz covariant under longitudinal boosts

$$
\tau=\sqrt{t^{2}-z^{2}}
$$

and a space-time rapidity variable

$$
\eta=\frac{1}{2} \ln \left(\frac{t-z}{t+z}\right)
$$

For free streaming particles

$$
z=v t=\frac{p_{z}}{E} t
$$

we see that the space-time rapidity equals the momentum space rapidity

$$
\eta=y
$$

If we have distributions of particles which are slowly varying in rapidity, it should be a good approximation to take the distributions to be rapidity invariant. 


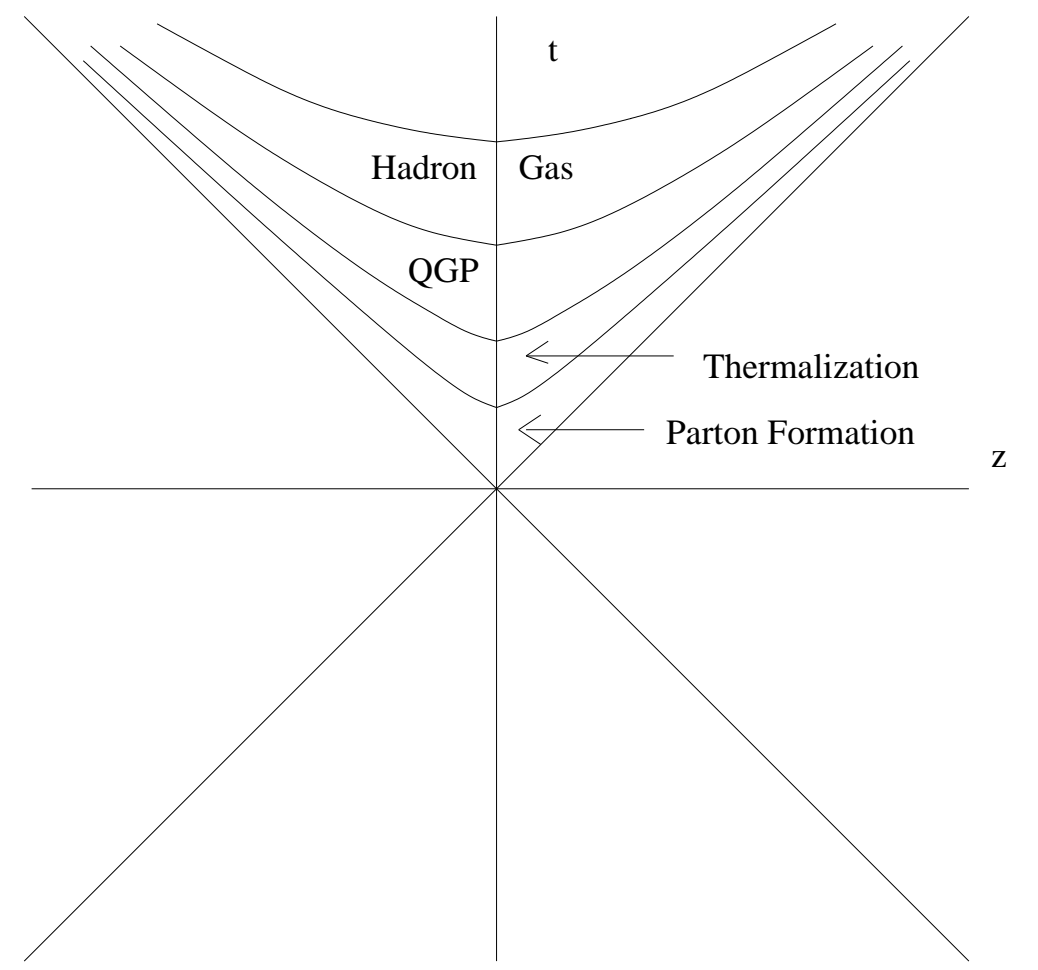

FIGURE 10. A space-time picture of ultrarelativistic nuclear collisions. 
This should be valid at very high energies in the central region. By the correspondence between space-time and momentum space rapidity, it is plausible therefore to assume that distributions are independent of $\eta$. Therefore distributions are the same on lines of constant $\tau$, which is as shown in Fig. 10. At $z=0, \tau=t$, so that $\tau$ is a longitudinally Lorentz invariant time variable.

We expect that at very late times, we have a free streaming gas of hadrons. These are the hadrons which eventually arrive at our detector. At some earlier time, these particle decouple from a dense gas of strongly interacting hadrons. As we proceed earlier in time, at some time there is a transition between a gas of hadrons and a plasma of quarks and gluons. This may be through a first order phase transition where the system might exist in a mixed phase for some length of time, or perhaps there is a continuous change in the properties of the system

At some earlier time, the quarks and gluons of the quark-gluon plasma are formed. This is at some time of the order of a Fermi, perhaps as small as .1 Fermi. As they form, the particles scatter from one another, and this can be described using the methods of transport theory. At some later time they have thermalized, and the system can be approximately described using the methods of perfect fluid hydrodynamics.

In the time between that for which the quarks and gluons have been formed and $\tau=0$, the particles are being formed. This is where the initial conditions are made.

In various levels of sophistication, one can compute the properties of matter made in heavy ion collisions at times later than the formation time. The problems are understood in principle for $\tau \geq \tau_{\text {formation }}$ if perhaps not in fact. Very little is known about the initial conditions.

In principal, understanding the initial conditions should be the simplest part of the problem. At the initial time, the degrees of freedom are most energetic and therefore one has the best chance to understand them using weak coupling methods in QCD.

There are two separate classes of problems one has to understand for the initial conditions. First the two nuclei which are colliding are in single quantum mechanical states. Therefore for some early time, the degrees of freedom must be quantum mechanical. This means that

$$
\Delta z \Delta p_{z} \geq 1
$$

Therefore classical transport theory cannot describe the particle down to $\tau=0$ since classical transport theory assumes we know a distribution function $f(\vec{p}, \vec{x}, t)$, which is a simultaneous function of momenta and coordinates. This can also be understood as a consequence of entropy. An initial quantum state has zero entropy. Once one describes things by classical distribution functions, entropy has been produced. Where did it come from?

Another problem which must be understood is classical charge coherence. At very early time, we have a tremendously large number of particles packed into a 
longitudinal size scale of less than a fermi. This is due to the Lorentz contraction of the nuclei. We know that the particles cannot interact incoherently. For example, if we measure the field due to two opposite charge at a distance scale $r$ large compared to their separation, we know the field fall as $1 / r^{2}$, not $1 / r$. On the other hand, in cascade theory, interactions are taken into account by cross sections which involve matrix elements squared. There is no room for classical charge coherence.

There are a whole variety of problems one can address in heavy ion collisions such

- What is the equation of state of strongly interacting matter?

- Is there a first order QCD phase transition?

These issues and others would take us beyond the scope of these lectures. The issues which I would like to address are related to the determination of the initial conditions, a problem which can hopefully be addressed using weak coupling methods in QCD.

\section{F Universality}

There are two separate formulations of universality which are important in understanding small $\mathrm{x}$ physics.

The first is a weak universality. This is the statement that physics should only depend upon the variable

$$
\Lambda^{2}=\frac{1}{\pi R^{2}} \frac{d N}{d y}
$$

As discussed above, this universality has immediate experimental consequences which can be directly tested.

The second is a strong universality which is meant in a statistical mechanical sense. At first sight it appear a formal idea with little relation to experiment. If it is however true, its consequences are very powerful and far reaching. What we shall mean by strong universality is that the effective action which describes small $\mathrm{x}$ distribution function is critical and at a fixed point of some renormalization group. This means that the behavior of correlation functions is given by universal critical exponents, and these universal critical exponents depend only on general properties of the theory such as the symmetries and dimensionality.

Since the correlation functions determine the physics, this statement says that the physics is not determined by the details of the interactions, only by very general properties of the underlying theory!

We can see how a renormalization group arises. In Fig 11, the space-time distribution of gluons is shown. The coordinate space rapidity is used. The effective action which we shall develop in later lectures is valid only for gluons with rapidity less than $\eta_{0}$. [5]- [8] Those at larger rapidity have been integrated out of the theory and appear only as recoilless sources of color charge for $\eta_{0} \leq \eta \leq y_{\text {proj }}$. 


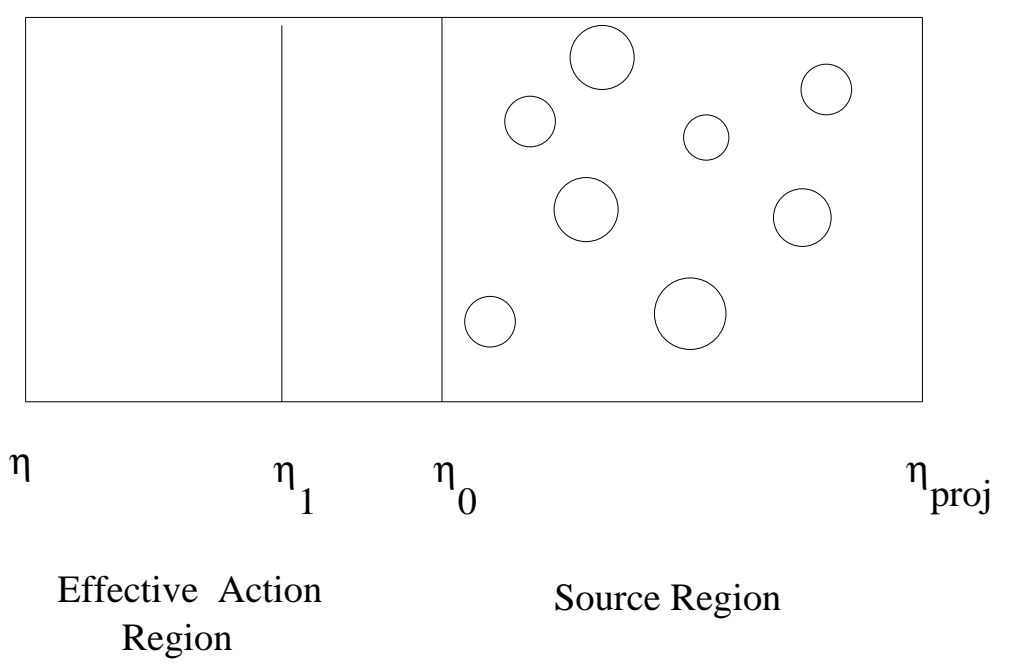

FIGURE 11. The space-time distribution function for glue inside of a hadron.

The way a renormalization group is generated is by integrating out the gluon degrees of freedom in the range $\eta_{1} \leq \eta \leq \eta_{o}$, to generate a new effective action for rapidity $\eta \leq \eta_{1}$. We can show that this results in new source strength of color charge, now in the range $\eta_{1} \leq \eta \leq y_{\text {frag }}$, and in a modification of some of the coefficients of the effective action.

At high $Q^{2}$, the renormalization group analysis simplifies, and one can show that in various limits reduces to the BFKL or DGLAP analysis. The renormalization group equations at smaller $q^{2}$ become more complicated, and have yet to be written in explicit form and evaluated. This is in principle possible to do. [9]

An essential ingredient in this analysis is the appearance of an action and of a classical gluon field. We should expect the appearance of a classical gluon field when the phase space density of gluons becomes high. Gluons are after all bosons, and when the phase space density is large they should be described classically. This provides the essential difference between older renormalization group analysis which was formulated in terms of a distribution function. High density and its complications due to coherence require the introduction of a field. The new renormalization group analysis is phrased in terms of the effective action for the classical gluon field and jumps from an ordinary equation to a functional equation.

The classical gluon fields which we shall find are the non-abelian generalization of the Lienard-Wiechart potentials of electrodynamics. If we use the coordinate space variable $x^{-}$and realize that the source for the gluons arise from much smaller $x^{-}$than that at which we make the measurements, since they arise from gluons of much higher longitudinal momenta which are more Lorentz contracted, then the sources can be imagined as arising from a $\delta\left(x^{-}\right)$. The Lienard-Wiechart potentials also are proportional to $\delta\left(x^{-}\right)$, and so exist only in the sheet. The fields are also transversely polarized 


$$
B_{a}^{i} \perp E_{a}^{i} \perp \hat{z}
$$

\section{G Why an Effective Action?}

The effective action formalism which will be advocated in the next lectures is very powerful. It is used to compute a gluon effective field. This field can be related to the wavefunction of the hadron.

This field allows one to generalize it from the original description of a single hadron, to collisions of hadrons and also to diffractive processes. We shall see that the effective action formulation is incredibly powerful.

The careful student might at this point be very worried: How has the problem been in any way simplified? We have just introduced a source, and to specify the field one has to specify the source. Moreover, such a specification is gauge dependent. What happens is amusing: We integrate over the all color orientations of the source. Gauge invariance is restored by the integration. The theory becomes specified by the local density of color charge squared. This is a gauge invariant quantity. It can be related to the gluon distribution functions, and is determined by the renormalization group equations.

\section{AN INTRODUCTION TO LIGHT CONE PHYSICS}

This lecture will provide an introduction to light cone kinematics and quantization of field theory on the light cone. We will eventually use light cone methods to quantize QCD, using the light cone gauge.

Light cone coordinates are

$$
x^{ \pm}=\frac{1}{\sqrt{2}}\left(x^{0} \pm x^{3}\right)
$$

and momenta

$$
p^{ \pm}=\frac{1}{\sqrt{2}}\left(p^{0} \pm p^{3}\right)
$$

The invariant dot product is

$$
p \cdot x=p_{t} \cdot x_{t}-p^{+} x^{-}-p^{-} x^{+}
$$

where $p_{t}$ and $x_{t}$ are transverse coordinates. This implies that in this basis the metric is $g^{+-}=g^{-+}=-1, g^{i j}=\delta^{i j}$ where $i, j$ refer to transverse coordinates. All other elements of the metric vanish.

An advantage of light cone coordinates is that if we do a Lorentz boost along the longitudinal direction with Lorentz gamma factor $\gamma=\cosh (y)$ then $p^{ \pm} \rightarrow e^{ \pm y} p^{ \pm}$ 


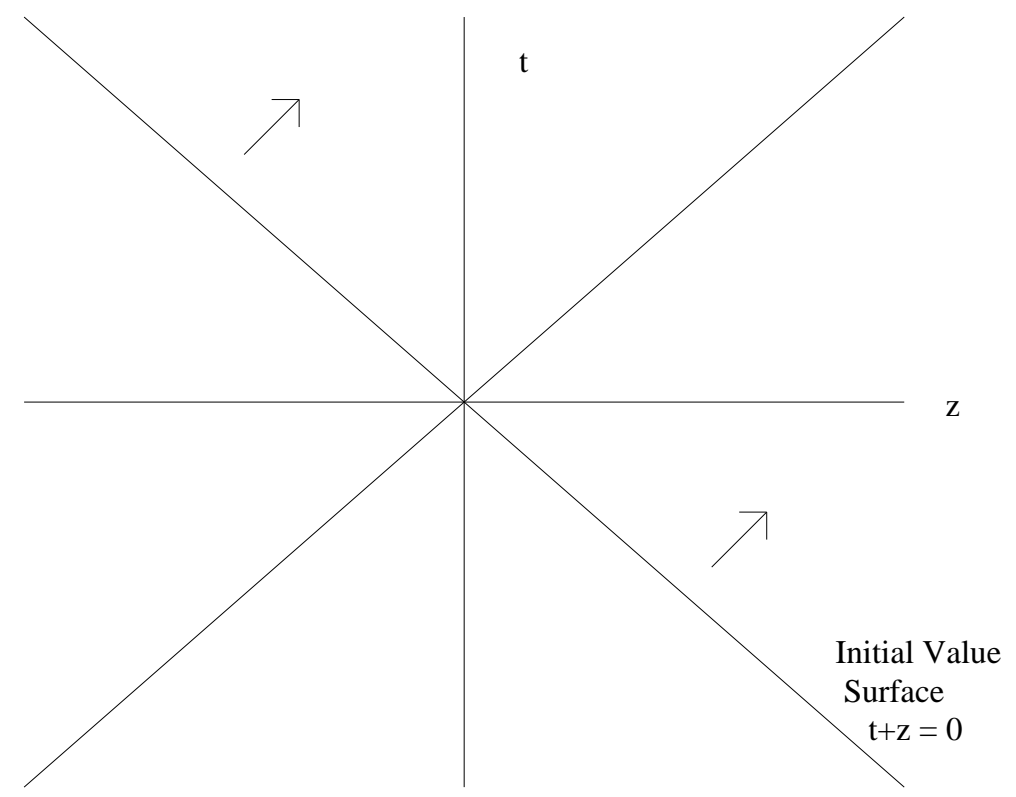

FIGURE 12. The initial data problem on the light cone.

If we let $x^{+}$be a time variable, we see that the variable $p^{-}$is to be interpreted as an energy. Therefore when we have a field theory, the component of the momentum operator $P^{-}$will be interpreted as the Hamiltonian. The remaining variables are to be thought of as momenta and spatial coordinates. In Fig. 12, there is a plot of the $z, t$ plane. The line $x^{+}=0$ provides a surface where initial data might be specified. Time evolution is in the direction normal to this surface.

We see that an elementary wave equation

$$
\left(p^{2}+M^{2}\right) \phi=0
$$

is particularly simple in light cone gauge. Since $p^{2}=p_{t}^{2}-2 p^{+} p^{-}$this equation is of the form

$$
p^{-} \phi=\frac{p_{t}^{2}+M^{2}}{2 p^{+}} \phi
$$

is first order in time. In light cone coordinates, the dynamics looks similar to that of the Schrodinger equation. The initial data to be specified is only the value of the field on the initial surface.

In the conventional treatment of the Klein-Gordon field, one must specify the field and its first derivative (the momentum) on the initial surface. In the light cone coordinate, the field is sufficient and the field momentum is redundant. This means that the field momentum will not commute with the field on the initial time surface!

Lets us work all this out with the example of the Klein Gordon field. The action for this theory is 


$$
S=-\int d^{4} x\left\{\frac{1}{2}(\partial \phi)^{2}+\frac{1}{2} M^{2} \phi^{2}\right\}
$$

The field momentum is

$$
\Pi\left(x_{t}, x^{-}\right)=\frac{\delta S}{\delta \partial_{+} \phi}=\partial_{-} \phi=\frac{\partial}{\partial x^{-}} \phi
$$

Note that $\Pi$ is a derivative of $\phi$ on the initial time surface. It is therefore not an independent variable, as would be the case in the standard canonical quantization of the scalar field.

We postulate the equal time commutation relation

$$
\left[\Pi\left(x_{t}, x^{-}\right), \phi\left(y_{t}, y^{-}\right)\right]=-i \delta^{(3)}(x-y)
$$

Here we time is $x^{+}=y^{+}=0$ in both the the field and field momentum. We see therefore that

$$
\partial_{-}[\phi(\vec{x}), \phi(\vec{y})]=-i \delta^{(3)}(x-y)
$$

or

$$
[\phi(x), \phi(y)]=-i \epsilon\left(x^{-}-y^{-}\right) \delta^{(2)}(x-y)
$$

Here $\epsilon(v)$ is $1 / 2$ for $v>0$ and $-1 / 2$ for $v<0$.

These commutation relations may be realized by the field

$$
\begin{aligned}
\phi(x) & =\int \frac{d^{3} p}{(2 \pi)^{3} 2 p^{+}} e^{i p x} a(p) \\
& =\int_{p^{+}>0} \frac{d^{3} p}{(2 \pi)^{3} 2 p^{+}}\left\{e^{i p x} a(p)+e^{-i p x} a^{\dagger}(p)\right\}
\end{aligned}
$$

Using

$$
\left[a(p) \cdot a^{\dagger}(q)\right]=2 p^{+}(2 \pi)^{3} \delta^{(3)}(p-q)
$$

the student can verify that the equal time commutation relations for the field are satisfied.

The quantity $1 / p^{+}$in the expression for the field in terms of creation and annihilation operators is singular when $p^{+}=0$. When we use a principle value prescription, we reproduce the form of the commutation relations postulated above with the factor of $\epsilon\left(x^{-}-y^{-}\right)$. Different prescriptions correspond to different choices for the inversion of $\frac{1}{\partial^{-}}$. One possible prescription is the Liebrandt-Mandelstam prescription $1 / p^{+}=p^{-} /\left(p^{+} p^{-}+i \epsilon\right)$. This prescription has some advantages relative to the principle value prescription in that it maintains causality at intermediate stages of computations and the principle value prescription does not. In the end, 
for physical quantities, the choice of prescription cannot result in different results. Of course, in some schemes the computations may become prohibitively difficult.

The student should now check that with the field above, the light cone Hamiltonian is

$$
P^{-}=\int_{p^{+}>0} \frac{d^{3} p}{(2 \pi)^{3} 2 p^{+}} \frac{p_{t}^{2}+M^{2}}{2 p^{+}} a^{\dagger}(p) a(p)
$$

as it must be.

In a general interacting theory, the Hamiltonian will of course be more complicated. The representation for the fields in terms of creation and annihilation operators will be the same as above. Note that all particles created by a creation operator have positive $P^{+}$. Therefore, since the vacuum has $P^{+}=0$, there can be no particle content to the vacuum. It is a trivial state. Of course this must be wrong since the physical vacuum must contain condensates such as the one responsible for chiral symmetry restoration. It can be shown that such non-perturbative condensates arise in the $P^{+}=0$ modes of the theory. We have not been careful in treating such modes. For perturbation theory, presumably to all orders, the above treatment is sufficient for our purposes.

\section{A Light Cone Gauge QCD}

In QCD we have a vector field $A_{a}^{\mu}$. This can be decomposed into longitudinal and transverse parts as

$$
A_{a}^{ \pm}=\frac{1}{\sqrt{2}}\left(A_{a}^{0} \pm A^{z}\right)
$$

and the transverse as lying in the tow dimensional plane orthogonal to the beam $\mathrm{z}$ axis. Light cone gauge is

$$
A_{a}^{+}=0
$$

In this gauge, the equation of motion

$$
D_{\mu} F^{\mu \nu}=0
$$

is for the + component

$$
D_{i} F^{i+}-D^{+} F^{-+}=0
$$

which allows one to compute $A^{-}$in terms of $A^{i}$ as

$$
A^{-}=\frac{1}{\partial^{+2}} D^{i} \partial^{+} A^{i}
$$


This equation says that we can express the longitudinal field entirely in terms of the transverse degrees of freedom which are specified by the transverse fields entirely and explicitly. These degrees of freedom correspond to the two polarization states of the gluons.

We therefore have

$$
A_{a}^{i}(x)=\int_{p^{+}>0} \frac{d^{3} p}{(2 \pi)^{3} 2 p^{+}}\left(e^{i p x} a_{a}^{i}(p)+e^{-i p x} a_{a}^{i \dagger}(p)\right)
$$

where

$$
\left[a_{a}^{i}(p), a_{b}^{\mathrm{J}^{\dagger}}(q)\right]=2 p^{+} \delta_{a b} \delta^{i j}(2 \pi)^{3} \delta^{(3)}(p-q)
$$

where the commutator is at equal light cone time $x^{+}$.

\section{B Distribution Functions}

We would like to explore some hadronic properties using light cone field operators. For example, suppose we have a hadron and ask what is the gluon content of that hadron. Then we would compute

$$
\frac{d N_{\text {gluon }}}{d^{3} p}=<h\left|a^{\dagger}(p) a(p)\right| h>
$$

The quark distribution for quarks of flavor $i$ (for the sum of quarks and antiquarks) would be given in terms of creation and annihilation operators for quarks as

$$
\frac{d N_{i}}{d^{3} p}=<h\left|\left\{b_{i}^{\dagger}(p) b_{i}(p)+d_{i}^{\dagger}(p) d_{i}(p)\right\}\right| h>
$$

where $b$ corresponds to quarks and $d$ to antiquarks. The creation and annihilation operators for quarks and gluons can be related to the quark coordinate space field operators by techniques similar to those above. The interested student should read the notes of Venugopalan for details. [10]

How would we begin computing such distribution functions? We will start with the example of a large nucleus, as this makes some issues conceptually simpler. We will then generalize to hadrons, where we shall see that the ideas presented here have a generalization.

For a large nucleus, we assume that the gluon distribution which we shall try to compute has longitudinal momentum soft compared to that of the valence quarks. Valence quarks have longitudinal momentum of the order of that of the nucleus, so this requirement is only that $x<<1$ In fact, we will require that $x<<A^{-1 / 3}$. This is the requirement that in the frame where the longitudinal momentum of the gluons is zero, the nucleus has a Lorentz contracted size much less than the wavelength associated with the gluons transverse momentum $\lambda \sim 1 / p_{T}$. This is 


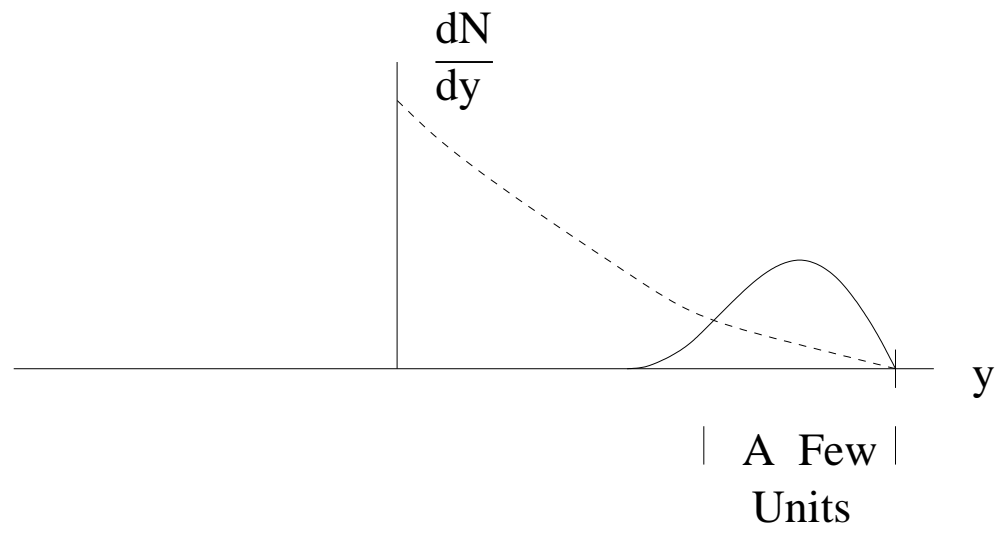

FIGURE 13. The rapidity distribution for quarks and gluons inside of a hadron.

the requirement that the gluon resolve the nucleus as a whole and is insensitive to the details of the nuclear structure (spatial distribution of valence quarks within the nucleus).

In Fig. 13, a rapidity distribution for gluons and for valence quarks is shown. We are interested in the region where the overlap between the quark and gluon rapidity distribution is small.

If the gluon phase space density is very large, the quantum gluons of the nucleus may be treated as classical fields. This may be true if

$$
\Lambda^{2}=\frac{1}{\pi R^{2}} \frac{d N_{\text {glue }}}{d y}
$$

satisfies $\Lambda^{2}>>\Lambda_{Q C D}^{2}$. Certainly if the typical $p_{T}$ of the gluons was of order $\Lambda_{Q C D}$ this would be true since the gluons would then be closely packed together. We shall see that this is true in fact for gluons with $p_{T}<<\Lambda$ which for high gluons density can become very large.

If the valence quarks have a longitudinal momentum much larger than the typical gluon momentum then their typical interactions with these gluons should be characterized by the soft momentum scale (otherwise the soft gluons would not remain soft). In an emission of a gluon, the emitted gluon has momentum very small compared to the valence quark longitudinal momentum. Its velocity therefore is barely changed by this emission. The quarks are therefore recoilless sources of color charge for the gluon classical field.

The picture we have is therefore that of Fig. 14. The valence quarks are sources for the gluons and sit on a sheet of thickness infinitesimal compared to the typical wavelength associated with the gluon field. We shall further assume for simplicity that the transverse distribution of charge is uniform. (In fact, a better approximation is that the distribution of charge is slowly varying on the gluon wavelength scale of interest. This case can be computed directly from knowledge of the uniform transverse distribution case, The transverse size scale of variation 


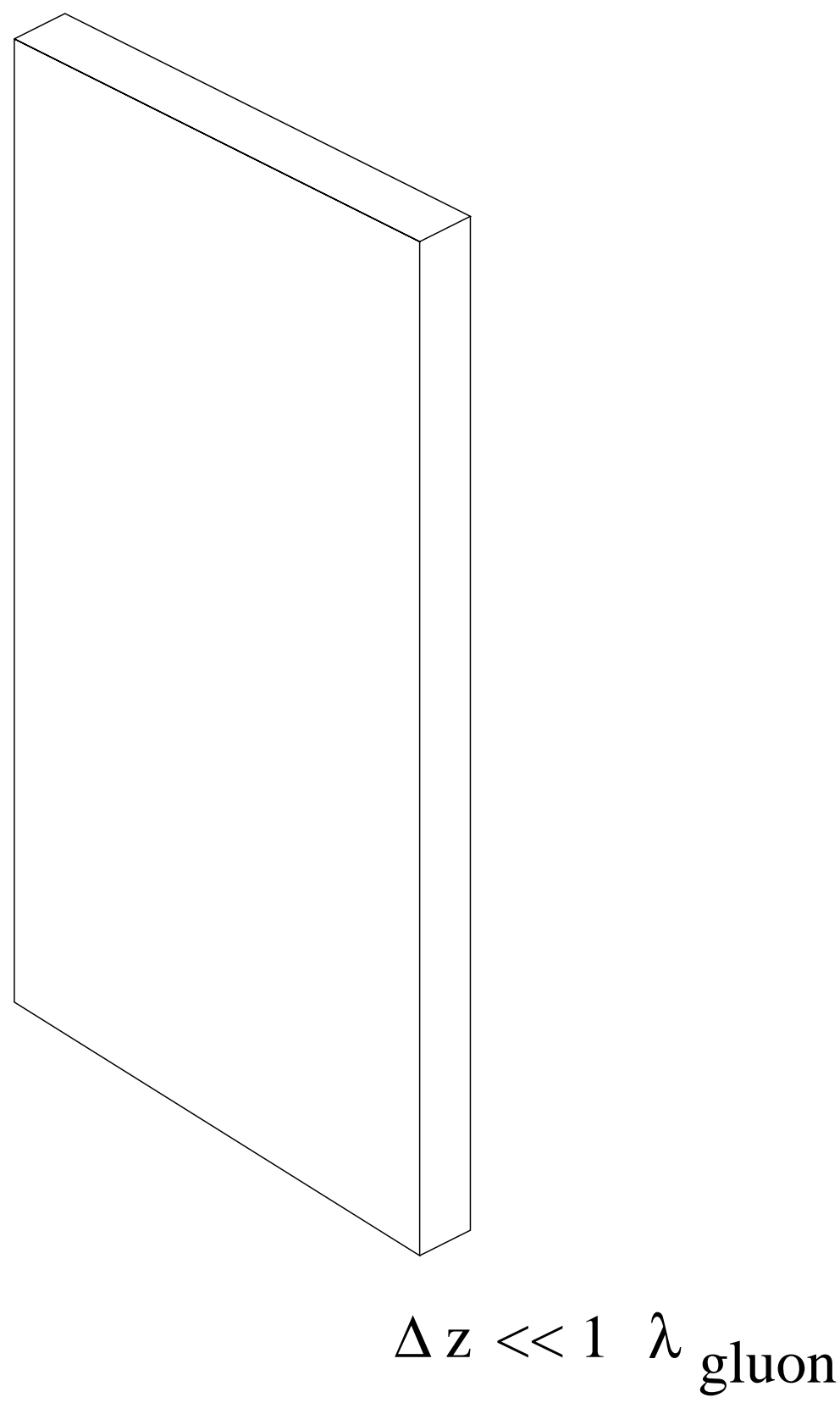

FIGURE 14. The nucleus as a source of charge sitting on a Lorentz contracted sheet. 


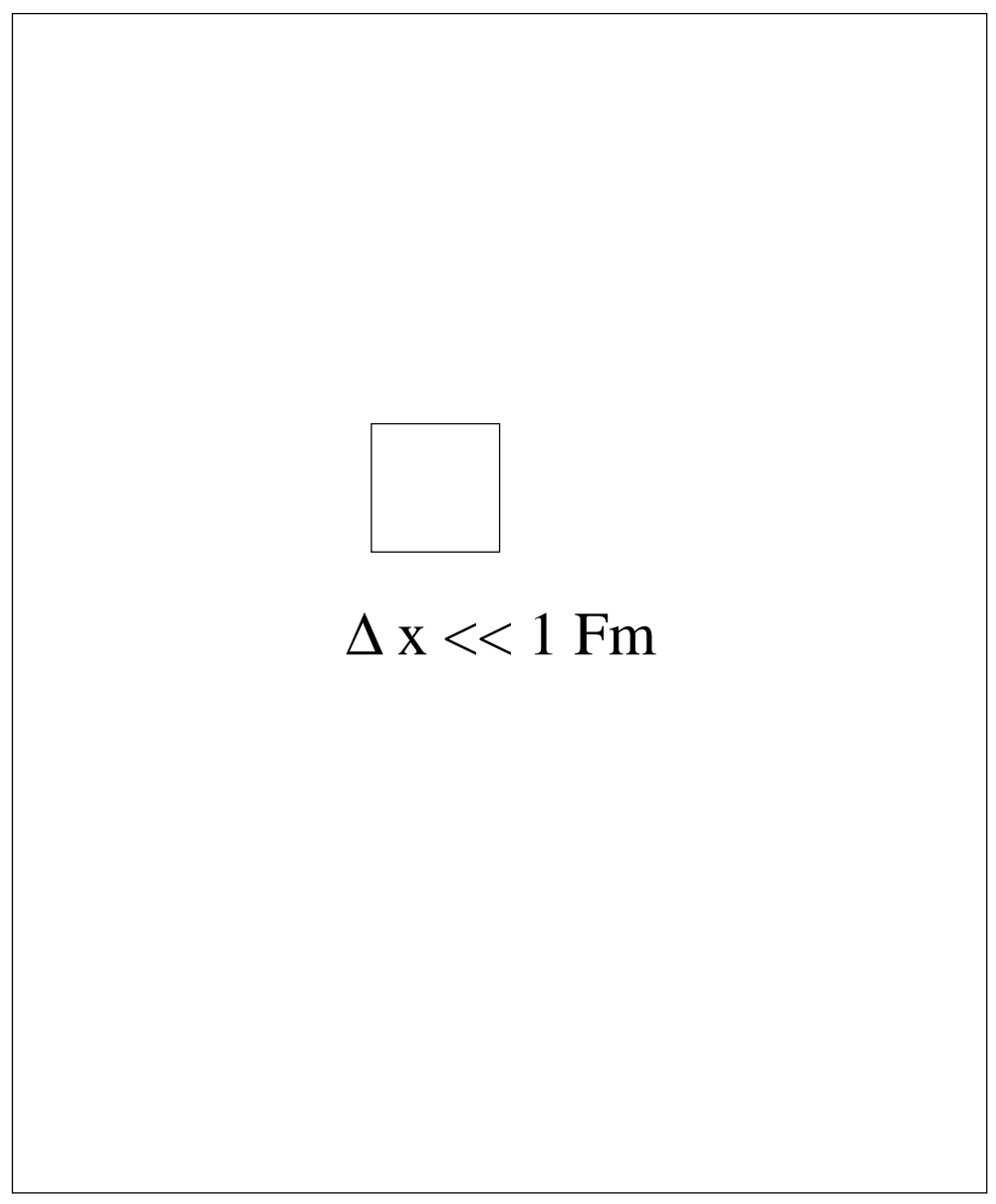

FIGURE 15. A head on picture of a nucleus.

is the nuclear radius, which is much larger than a fermi, so that this criteria is satisfied so long as $\Lambda>>\Lambda_{Q C D}$, and the typical transverse momentum is of order A.)

In Fig. 15, a head on picture of the nucleus is shown. A small square is indicated which shows the transverse resolution size scale that we shall employ to probe the nucleus. In order that we can use weak coupling methods, we require that the transverse size be $\Delta x<<1 F m$.

In Fig. 16, the nucleus is shown along the beam axis. Where the tube generated by the square associated with $\Delta x$ intersects a quark or gluons shown an $\mathrm{x}$. (We use space-time rapidity variables to spread out the nucleus. The precise definition of this variable is below. For now, just imagine that we have chosen a longitudinal spatial variable which spreads out the Lorentz contracted hadron.) The physical extent in longitudinal spatial coordinate is of course small. If we require that $\Delta x<<1 F m$ and require that a tube intersects a quark or gluon from some hadron, then typically, that hadron will be far separated from any other hadron which 


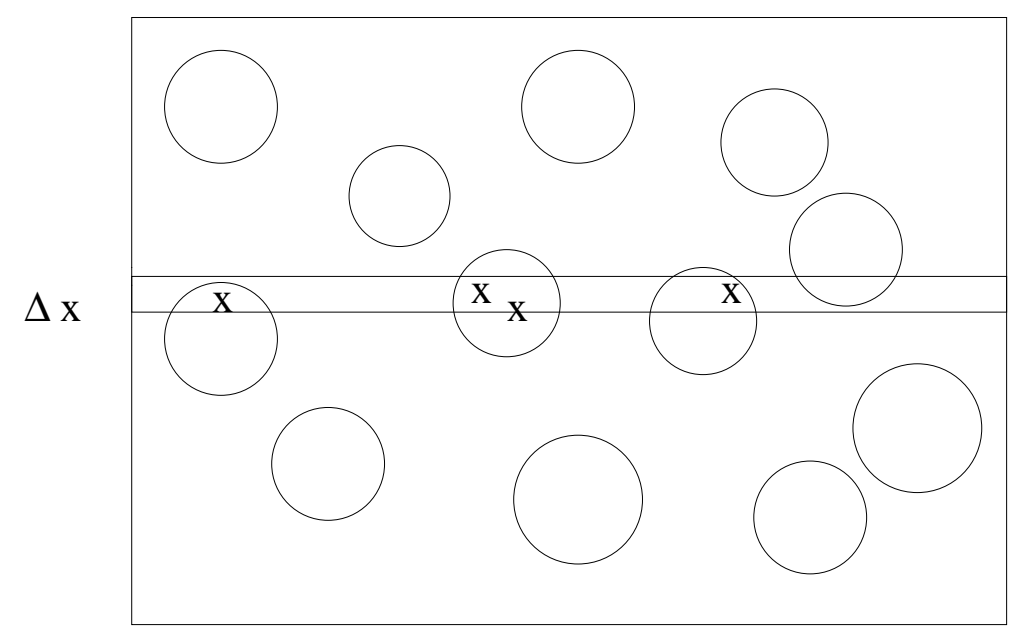

FIGURE 16. The nucleus as seen longitudinally with the longitudinal axis blown up by a choice of variable such as rapidity.

has one of its quarks or gluons within the tube. The quarks and gluons within the tube are therefore uncorrelated in color. If we further require that the tube be large enough so that many quarks and gluons are in each tube, a situation always possible if we have large enough energy or a large enough nucleus, then the color total color charge associated with these quarks and gluons will be in a high dimensional representation, and can be treated classically. To see this, recall that in a high dimensional representation, $Q^{2}>>\sim[Q, Q]$ so that commutators may be ignored.

For partons at the $\mathrm{x}$ values where we wish to compute the gluon distribution function, the higher rapidity sources sit on a sheet of infinitesimal thickness. The problem we must solve is to compute the typical correlation functions which give distribution functions for stochastic sources sitting on a sheet of infinite transverse extent traveling at the speed of light. Mathematically, this problem is similar to spin glass problems in condensed matter physics. Such problems typically have entirely nontrivial renormalization groups, and we will see that this is the case for our problem.

To understand a little better how the sources are distributed, it is useful to go inside the sheet. This is accomplished by introducing a space-time rapidity variable. Let us assume that the rapidity of the projectile is $y_{\text {proj }}$ and its longitudinal momentum is $P^{+}$, we define

$$
\eta=y_{p r o j}-\ln \left(P_{p r o j}^{+} x^{-}\right)
$$

We define the momentum space rapidity as

$$
y=y_{\text {proj }}-\ln \left(P_{\text {proj }}^{+} / p^{+}\right)
$$

Here $x^{-}$is the coordinate of a source and $p^{+}$its momentum. The previous definition of momentum space rapidity for produced particles was 


$$
y=\frac{1}{2} \ln \left(p^{+} / p^{-}\right)=\ln \left(p^{+} / m_{t}\right)
$$

where $m_{t}=\sqrt{p_{t}^{2}+m^{2}}$. This last expression is valid for particles on mass shell, whereas the other definition of momentum space rapidity works for constituents of the hadron wavefunction, which are of course not on mass shell. These definitions are equal within about a unit of rapidity for typical values of transverse momenta. In the wavefunction, the uncertainty principle relation gives $x^{-} p^{+} \sim 1$, so that we see the space time rapidity is up to about a unit of rapidity the same as both momentum space values. We will therefore use these rapidities interchangeably in what follows.

We can now implement our static sources of charge by assuming the theory is defined by an ensemble of such charges:

$$
Z=\int[d \rho] \exp \left\{-\frac{1}{2} \int_{y}^{y_{p r o j}} d y^{\prime} d^{2} x_{t} \frac{1}{\mu^{2}\left(y^{\prime}\right)} \rho^{2}\left(y^{\prime}, x_{t}\right)\right\}
$$

This ensemble gives

$$
<\rho^{a}\left(y, x_{T}\right) \rho^{b}\left(y^{\prime}, x_{t}^{\prime}\right)>=\mu^{2}(y) \delta^{a b} \delta\left(y-y^{\prime}\right) \delta^{(2)}\left(x_{t}-x_{t}^{\prime}\right)
$$

The parameter $\mu^{2}$ therefore has the interpretation of a charge squared per unit rapidity

$$
\mu^{2}(y)=\frac{1}{N_{c}^{2}-1} \frac{1}{\pi R^{2}} \frac{d Q^{2}(y)}{d y}
$$

The total charge squared is

$$
\chi(y)=\int_{y}^{y_{p r o j}} d y^{\prime} \mu^{2}\left(y^{\prime}\right)
$$

Since the sources are individual quarks and gluons, this can also be related directly to the total number of gluons and quarks contributing to the source as [11]

$$
\chi(y)=\frac{1}{\pi R^{2}}\left(\frac{N_{g}}{2 N_{c}}+\frac{N_{c} N_{q}}{N_{c}^{2}-1}\right) \int_{x}^{1} d x G(x)
$$

The factors above arise from computing the color charge squared of a singe quark or a single gluon.

We may now construct the non-abelian Lienard-Wiechart potentials generated by this distribution of sources. We must solve the equation

$$
D_{\mu} F^{\mu \nu}=g^{2} \delta^{\nu+} \rho\left(y, x_{t}\right)
$$

To solve this equation, we look for a solution of the form 


$$
A^{ \pm}=0
$$

and

$$
A^{i}=\frac{1}{i} U\left(y, x_{t}\right) \nabla^{i} U^{\dagger}\left(y, x_{T}\right)
$$

We could equally well solve this equation in a gauge where $A^{+}$is nonzero and all other components vanish. The student should check that the gauge transformation induced by $U$ gives

$$
\bar{A}^{+}=\frac{1}{i} U^{\dagger} \partial^{+} U
$$

In this non-lightcone gauge, the field equations simplify and we get

$$
\nabla_{t}^{2} \bar{A}^{+}=g^{2} \rho\left(y, x_{t}\right)
$$

Solving for $U$ gives

$$
U=\exp \left\{i \int_{y}^{y_{\text {proj }}} d y^{\prime} \frac{1}{\nabla_{t}^{2}} \rho\left(y^{\prime}, x_{t}\right)\right\}
$$

We have therefore constructed an explicit expression for the light cone field $A^{i}$ in terms of the source $\rho$ for arbitrary $\rho$ ! The system is integrable.

The structure of the fields strengths $E$ and $B$ which follow from this field strength is now easy to understand. The only nonzero longitudinal derivative is $\partial^{+}$. The field strength $F_{i j}$ where both $i j$ are transverse vanishes since the filed looks like a pure gauge transformation in the two dimensional space. It is in fact a pure gauge everywhere but in the sheet where the charge sits. Therefore the only nonvanishing field strength is $F^{i+}$. This gives $E \perp B \perp \vec{z}$, that is the fields are transversely polarized. This is the precise analog of the Lienard-Wiechart potentials of electrodynamics.

\section{LECTURE 3: THE ACTION AND RENORMALIZATION GROUP}

The effective action for the theory we have described must be gauge invariant and properly describe the dynamics in the presence of external sources, up to an overall gauge transformation which is constant in $x^{ \pm}$. (The lack of precise gauge invariance arises from the the desire to define the intrinsic transverse momentum of gluon distribution functions. Although when used in computations of gauge dependent quantities, the gauge dependence disappears, it is useful to not have the full gauge invariance in intermediate steps of computation.) The student should verify, that consistency with the Yang-Mills equations in the presence of an external source requires that 


$$
D_{\mu} J^{\mu}=0
$$

For a source of the type we have here,

$$
J_{a}^{\mu}=\delta^{\mu a} \delta\left(x^{-}\right) \rho^{a}\left(x_{t}\right)
$$

This is an approximation we make when we describe the source on scales much larger than the spatial extent of the source. To properly regularize the delta function, we need to spread the source out in $x^{-}$as was done in the previous section. We find that the action is

$$
S=-\frac{1}{4} \int d^{4} x F_{\mu \nu}^{a} F_{a}^{\mu \nu}+\frac{i}{N_{c}} \int d^{2} x_{t} d x^{-} \delta\left(x^{-}\right) \rho^{a}\left(x_{t}\right) \operatorname{tr} T^{a} \exp \left\{i \int_{-\infty}^{\infty} d x^{+} T \cdot A^{-}(x)\right\}
$$

In this equation, the matrix $T$ is in the adjoint representation of the gauge group. This is required for reality of the action. The student should minimize this action to get the Yang-Mills equations, identify the current, and show that the current is covariantly conserved.

This action is gauge invariant under gauge transformations which are required to be periodic in the time $x^{+}$. This is a consequence of the gauge invariance of the measure of integration over the sources $\rho$. This will be taken as a boundary condition upon the theory. In general if we had not integrated over sources, one could not define a gauge invariant source, as gauge rotations would change the definition of the source. Here because the source is integrated over in a gauge invariant way, the problem does not arise.

In the most general gauge invariant theory which we can write down is generated from

$$
Z=\int[d \rho] e^{-F[\rho]} \int[d A] e^{i S[A, \rho]}
$$

This is a generalization of the Gaussian ansatz described in the previous lecture. It allows for a slightly more complicated structure of stochastic variation of the sources. The Gaussian ansatz can be shown to be valid when the evaluating structure functions at large transverse momenta.

$$
F_{\text {Gaussian }}[\rho]=\frac{1}{2 \chi} \int d^{2} x_{t} \rho^{2}\left(x_{t}\right)
$$

This theory is a slight modification of what we described in the first lecture. We have here assumed that our theory is an effective theory valid only in a limited range of rapidity much less than the rapidity of the source. In this restricted range, the structure of the source in rapidity cannot be important, and therefore we couple only to the total charge seen at the rapidity of interest. The local charge density as a function of rapidity is replaced by the total charge at rapidities greater than that at which we measure the field. The scale of fluctuation of the source is instead of 
the local charge squared per unit are per unit rapidity $\mu^{2}(y)$ becomes replaces by the scale of fluctuation of the total charge. The student should prove that

$$
\chi=\int_{y}^{y_{p r o j}} d y^{\prime} \mu^{2}\left(y^{\prime}\right)
$$

To fully determine $F$ in the above equation demands a full solution of the renormalization group equations of the theory. This has yet to be done. We shall confine our attention in most of the analysis below to the Gaussian ansatz. It is remarkable that within this simple ansatz for $F$, most of the general feature which a full treatment should generate, such as proper unitary behavior of deep inelastic scattering, arises in a natural way.

Let us turn our attention for the moment to the gluon distribution function

$$
(2 \pi)^{3} 2 p^{+} \frac{d N}{d^{3} p}=<a^{\dagger}(p) a(p)>
$$

Using the results of the previous lecture, you should prove that

$$
\frac{d N}{d^{3} p}=\frac{2 p^{+}}{(2 \pi)^{3}} \sum_{i, a} D_{a a}^{i i}(p,-p)
$$

Here $a$ is a color index and $i$ is a transverse index associated with the gluon field. $D$ is the gluon propagator in the external field

$$
D_{a b}^{\mu \nu}(p, q)=<A_{a}^{\mu}(p) A_{b}^{\nu}(q)>
$$

(In field theory language, $D$ is the propagator in the external field including both connected and disconnected pieces.)

In lowest order, the $A$ in the expression for $D$ is simply the external LienardWiechart potential. Recall that

$$
A^{i}=-i U \nabla^{i} U^{\dagger}
$$

where the $U$ 's are the explicit functions of the source $\rho$ computed in the previous lecture.

The expectation value above may be computed using the Gaussian weight function. We need the propagator $1 / \nabla_{t}^{4}$ to perform this calculation. The student should try to do this computation (it is equivalent to normal ordering exponentials), and if it is too hard, refer to the paper of Jalilian-Marian et. al, where it is worked out in some detail. [7] The result is

$$
\frac{d N}{d^{2} p_{T}} \sim \int d^{2} z e^{-i p_{T} z_{T}} \frac{4\left(N_{c}^{2}-1\right)}{N_{c} z_{T}^{2}} \theta\left(1-z_{t} \Lambda_{Q C D}\right)\left\{1-\left(z_{t}^{2} \Lambda_{Q C D}^{2}\right)^{2 \pi \alpha_{s}^{2} \chi z_{t}^{2}}\right\}
$$

This results in the form for the gluon distribution function as shown in Fig. 17. At $p_{t}>>\Lambda$ where $\Lambda=\alpha_{s} \sqrt{\chi}$, the 


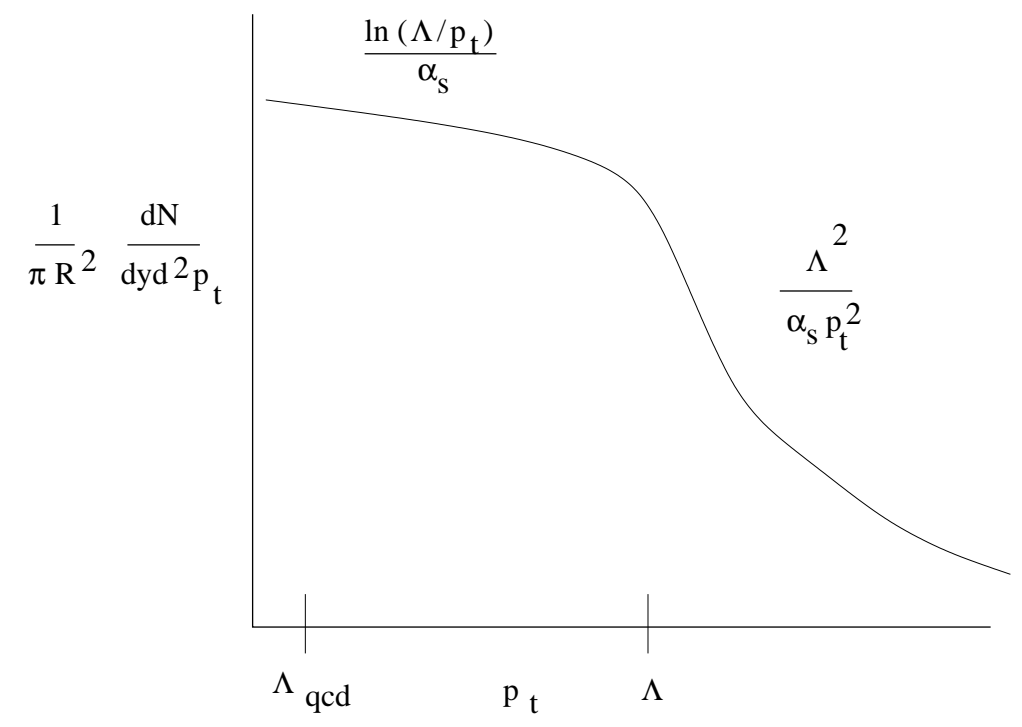

FIGURE 17. The intrinsic transverse momentum dependence of the gluon distribution function.

$$
\frac{1}{\pi R^{2}} \frac{d N}{d y d^{2} p_{t}} \sim \Lambda^{2} / \alpha_{s} p_{t}^{2}
$$

This is because this part of the distribution can be thought of as arising from bremstrahlung from the sources of the gluon field at high rapidities. As $p_{t} \leq \Lambda$, the gluon distribution function goes to a slowly varying function of $p_{T}$, which in the Gaussian ansatz is

$$
\frac{1}{\pi R^{2}} \frac{d N}{d y d^{2} p_{t}} \sim \frac{1}{\alpha_{s}} \ln \left(\Lambda / p_{t}\right)
$$

In general, we expect saturation in this region, for arguments which will be presented in the next few pages. This means we get a slowly varying function of $p_{T}$, which by dimensional arguments, is therefore a slowly varying function of $\Lambda$

The only place that any non-trivial rapidity dependence enters the problem is through $\Lambda$. This dependence can be found from the renormalization group analysis of the effective action. Therefore in the bremstrahlung region, the dependence on $\Lambda^{2}$ is linear, and in the saturation region it is very weak.

Let us assume that when we go beyond the Gaussian ansatz, the general features of saturation presented above remain. We will show that this results in a reasonable physical picture for small x physics, and solve the unitarity puzzle outlined in the first lecture. First consider the gluon distribution function. The total number of gluons which can be measured at some scale size larger than a resolution size scale $1 / Q^{2}$ is

$$
x G\left(x, Q^{2}\right)=\int_{0}^{Q^{2}} \frac{d^{2} p_{T}}{(2 \pi)^{2}} \frac{d N}{d y d^{2} p_{T}}
$$


For $Q^{2}>>\Lambda^{2}$, the integral is dominated by the bremstrahlung tail, and $G \sim$ $R^{2} \Lambda^{2}$. (The gluon distribution is proportional to the charge per unit area times the area.) In our random walk scenario, the effective charge squared must be proportional to the length of the random walk, $R$, so that $G \sim R^{3}$ which for a nucleus is proportional to $A^{1 / 3}$. This is what we expect at large $Q^{2}$, except the reasoning is a little different than usual. The standard argument would have been that at large $Q^{2}$, the degrees of freedom in a nucleus for example should act incoherently, and we should have the gluon distribution functions proportional to $A$. Here we have random fields generating precisely the same $A$ dependence!

When $Q^{2} \leq \Lambda^{2}$, the integral is dominated by the saturation region. Here $G \sim R^{2} Q^{2}$, and the gluons can be thought of as arising from the surface of the hadron. Again this is consistent with what is expected from phenomenology. The gluons are so soft that they cannot see the entire hadron, only its surface.

The effect of saturation for unitarization of deep inelastic scattering can also be easily understood. Suppose we are at some fixed $Q^{2}>>\Lambda^{2}(y)$. As $y$ decreases corresponding to going to smaller $x$, the gluon distribution function increases as $\Lambda^{2}(y)$. When $x$ becomes so small that we get into the saturation region, the linear $\Lambda$ dependence is weakened (in the Gaussian ansatz it is logarithmic in $\Lambda$ ), and the structure function stops growing. We expect that the number of gluons of size smaller than our resolution scale have ceased to grow, and the cross section should become slowly varying. This is in spite of the fact that $\Lambda$ continues to grow!

The physics is again simple to understand: At small $\mathrm{x}$ we are indeed adding more and more gluons to the hadron wavefunction. These gluons are smaller as their inverse size scales as gluon density,

$$
1 / r^{2} \sim \frac{1}{\pi R^{2}} \frac{d N}{d y}
$$

They do not contribute to a fixed $Q^{2}$ cross section when they become smaller than the resolution size scale. What saves unitarity is $p_{t}$ broadening.

\section{A Deep Inelastic Scattering}

In the previous discussion, we were concerned with the gluon distribution function. In deep inelastic scattering, we measure the quark distribution functions. How are these related?

In deep inelastic scattering we measure

$$
\begin{aligned}
& W^{m u \nu}=\frac{1}{2 \pi} \operatorname{Im} \int d^{4} x e^{i q x}<P_{h a d} \mid T\left(J^{\mu}(x) J^{\nu}(0) \mid P_{h a d}>\right. \\
& =\frac{1}{M_{h a d}}\left\{-\left(g^{\mu \nu}-\frac{q^{\mu} q^{\nu}}{q^{2}}\right) F_{1}+\right. \\
& \left.\left(p^{\mu}-q^{\mu} \frac{p \cdot q}{q^{2}}\right)\left(p^{\nu}-q^{\nu} \frac{p \cdot q}{q^{2}}\right) \frac{1}{p \cdot q} F_{2}\right\}
\end{aligned}
$$


We must be able to compute the imaginary part of the current-current correlation function. This is simply vacuum polarization in the presence of the LienardWiechart potentials. In fact, it is straightforward to compute the propagators in these background fields. [13] The reason why it is simple is because the background field is basically gauge transformations in two regions of spaces separated by a surface of discontinuity.

This propagator may be then used to compute the vacuum polarization. The computation can be done so far as to relate explicitly the structure functions to correlation functions of exponentials involving $\rho$ and expressions very similar to those for the gluon distribution function result. These results are currently being applied to the study of deep inelastic scattering to see whether the effects of saturation might be seen experimentally.

\section{B Renormalization Group and How It Works}

In the above computation of the gluon propagator, one used the classical effective action to compute the Lienard-Wiechart potentials. These classical fields were then used to compute the propogator. What about the quantum corrections?

The classical field contribution is shown in Fig 18. The $\mathrm{x}$ in the figure marks the position of the source. In Fig 19, the contribution arising by inserting the lowest order contribution to the connected piece of the gluon propogator is shown. Here there is a loop digram and the dashed line with the solid ellipse in it represents the gluon propogator to all orders in the strength of the external field. In Fig. 20, the piece where a quantum loop correction to one of the classical fields is shown.

When the quantum corrections are computed, one gets a correction to the classical field of order $\alpha_{s} \ln \left(x_{\text {cutoff } f} / x\right)$, where $x$ is the value for gluon distribution function, and $x_{\text {cutoff }}$ is the maximum $x$ appropriate for the effective action. Although the coupling is small, the logarithm can become big if we go to $x$ values far below the cutoff. The overall theory should remain valid however, the problem is that the classical approximation is no longer good.

The way to fix this up is to use the renormalization group. Suppose we want a new effective theory below $x_{n e w}$, and we require that $x_{n e w}<<x_{\text {cutoff }}$ but that

$\alpha_{s} \ln \left(x_{\text {cutoff }} / x_{\text {new }}\right)<<1$. This means that the quantum corrections are small in the range $x_{\text {new }}<<x<<x_{\text {cutoff }}$, and can be handled perturbatively. We proceed by integrating out the degrees of freedom in this intermediate range of $\mathrm{x}$, to generate a new effective theory in the region $x<<x_{n e w}$.

In this process, the one can show that the only thing that changes in the effective action formalism is the weight function $F[\rho]$ for fluctuations in the color source . Effectively, we are changing what we call source for the gluon field, and trading it for what we call the gluon field. This renormalization group procedure is that of Wilson-Kadanoff. One can derive the form of the renormalization group equations and they can be written explicitly in the low gluon density region. One can prove that here the function $F$ is Gaussian, and that in appropriate kinematic limits, 


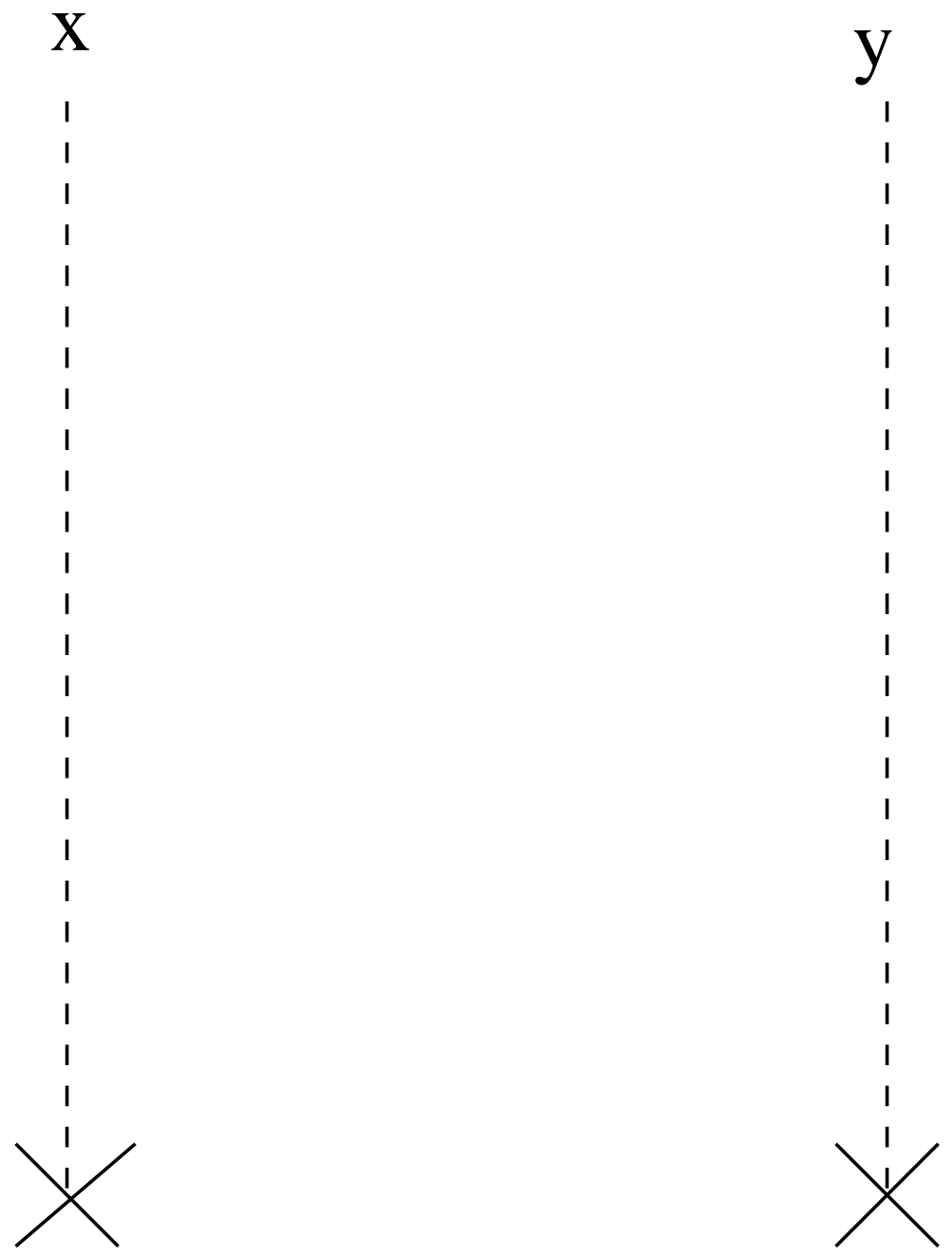

FIGURE 18. The classical field contribution to the gluon distribution function. 


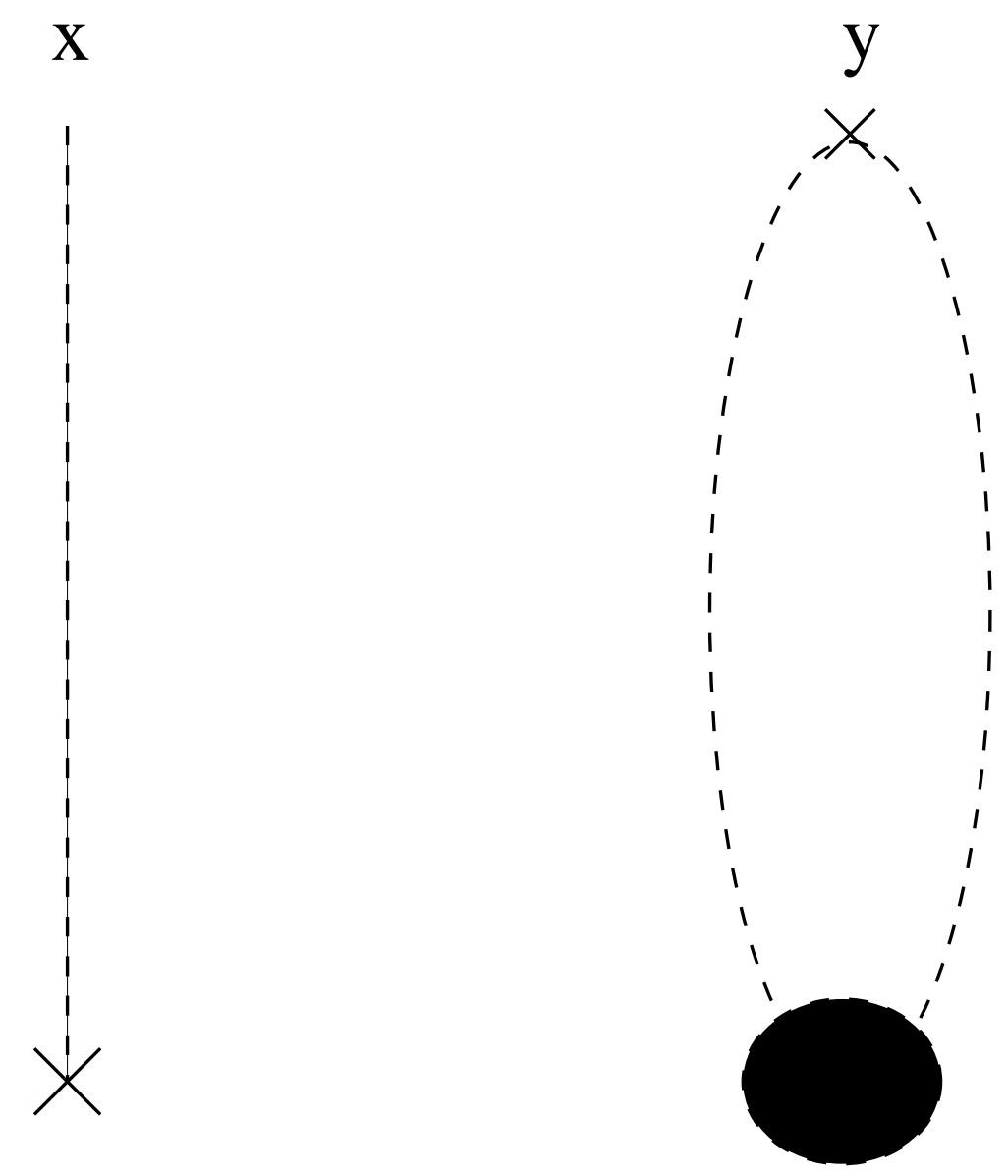

FIGURE 19. The first quantum correction to the classical field part of the gluon distribution function.

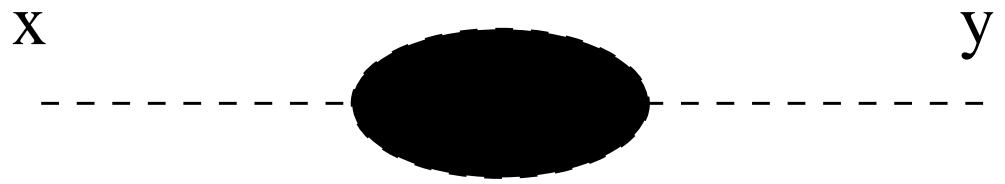

FIGURE 20. The quantum propogator correction to the gluon distribution function. 


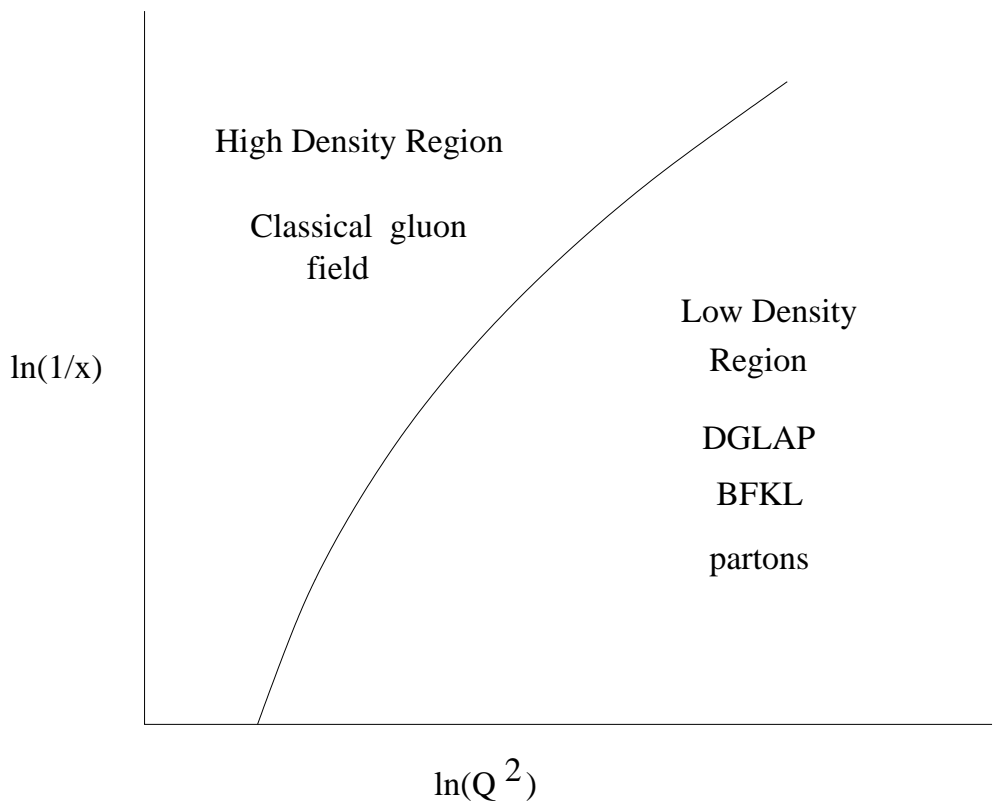

FIGURE 21. The $\ln (1 / x)$ and $\ln \left(Q^{2}\right)$ plane and various regions of high and low parton density.

one reproduces the BFKL equation, the DGLAP equation and their non-linear generalizations to first order in the non-linearities. Work is currently in progress to get the explicit form of these equations in the high density region, and to solve them.

The various regions of high and low density are shown in the plot of Fig. 21. If we are in the low density region, we evolve in $x$ by the BFKL equation and in $Q^{2}$ by the DGLAP equation. Hopefully a full solution to the problem will lead to an effective action which is at a fixed point of the renormalization group. In this case, at very small $\mathrm{x}$, the form for $F$ will simplify, and all correlation functions will have universal critical exponents. If this is true, the dynamics of high energy scattering for all hadrons becomes the same, and presumably has a simple structure.

\section{Other Directions}

In Raju Venugopalan's talk, you heard about using these techniques to describe high energy hadron-hadron collisions. [14]- [16] I have nothing to add to his talk, except that this shows that there is a direct relationship between hadron-hadron collisions and deep inelastic scattering.

A third class of phenomena is diffraction. In Fig. 22, a rapidity distribution of particle for a diffractive event is shown. There are two clusters of produced particles. In deep inelastic scattering these could be say the clump associated with a quark-anti-quark jet produced by the virtual photon, and a clump associated with the fragmentation of the target. If we restrict our attention to the class of diffractive 


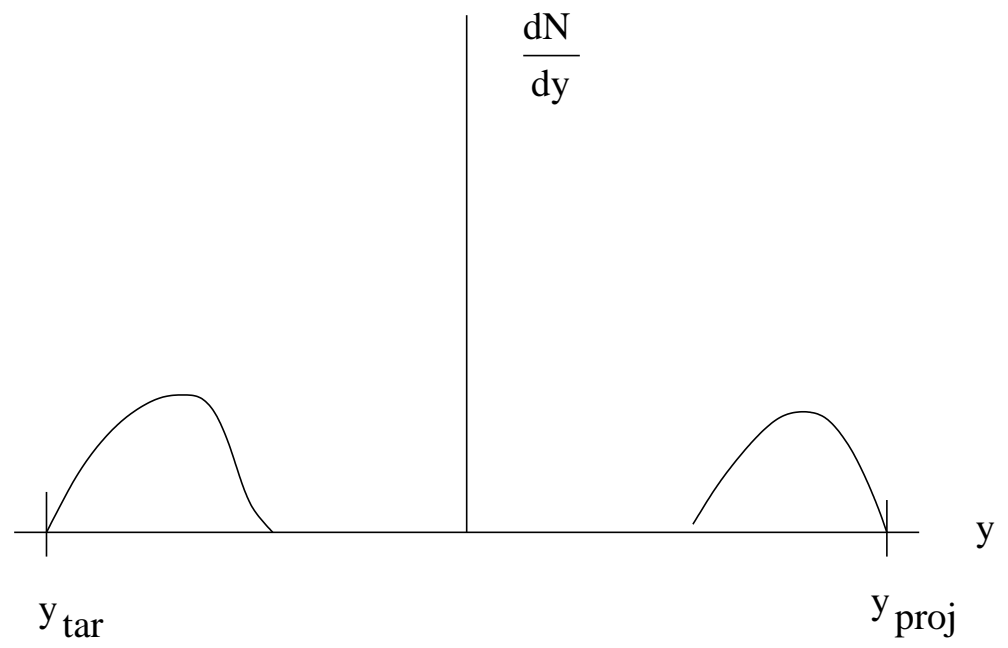

FIGURE 22. The rapidity distribution of particles produced in a diffractive event.

phenomenon where the target does not fragment, that is scatters elastically, one can prove the following: [17]- [19]

- Deep inelastic scattering is given by computing the amplitude for vacuum polarization for the electromagnetic current in the presence of the non-abelian classical field, squaring and then averaging over color.

- Diffractive events are given by computing the amplitude in the presence of the non-abelian color field, averaging over color, and then squaring.

We have argued the first case earlier in the lecture. To understand how the second case works, note that only the diagrams of Fig. 23 survive averaging over color before squaring the amplitude. In this figure a virtual photon produces a pair of quark jets. These jets exchange gluons (dash dot line) with the source which comprise the hadron. For sources are far away in rapidity from the jet, and therefore the longitudinal momentum transfer is very small. (The sources are not kicked by the jet. They do not change their velocity.) Moreover the color averaging and translational invariance guarantees that the transverse momentum given to the quarks after two scatterings vanishes.

So if we imagine a hadron and its Fock space constituents, they are unchanged by the scatterings shown in Fig. 23. Therefore the initial hadron state projects on to the hadron after scattering, in the amplitude, with weight very close to one, that is the hadron has scattered elastically (although many of its constituents have scattered.)

The amplitude shown in Fig. 23 therefore describes the rapidity distribution of particles shown in Fig. 22 only if the hadron does not fragment, that is, maintains its identity as a hadron. Therefore only one particle emerges on the hadron side of 


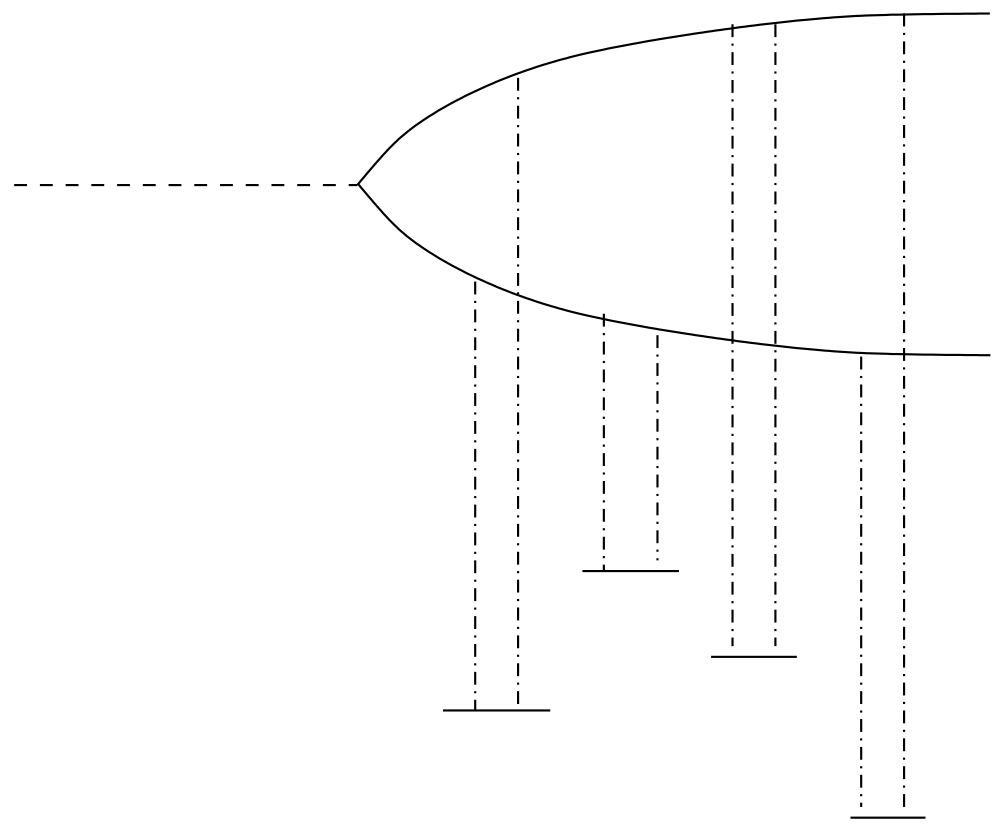

FIGURE 23. The non-zero contributions to the amplitude after color averaging.

the collision. It may be possible to generalize these considerations to fully diffractive processes where the hadron is allowed to fragment.

\section{ACKNOWLEDGMENTS}

I thank my colleagues Alejandro Ayala-Mercado, Miklos Gyulassy, Yuri Kovchegov, Alex Kovner, Jamal Jalilian-Marian, Andrei Leonidov, Raju Venugopalan and Heribert Weigert with whom the ideas presented in this talk were developed. This work was supported under Department of Energy grants in high energy and nuclear physics DOE-FG02-93ER-40764 and DOE-FG02-87-ER-40328. I particularly thank Andrei Leonidov for a critical reading of the manuscript.

\section{REFERENCES}

1. E.A. Kuraev, L.N. Lipatov and Y.S. Fadin, Zh. Eksp. Teor. Fiz 72, 3 (1977) (Sov. Phys. JETP 45, 1 (1977) ); I.A. Balitsky and L.N. Lipatov, Sov. J. Nucl. Phys. 28822 (1978); G. Altarelli and G. Parisi, Nucl. Phys. B126 298 (1977); Yu.L. Dokshitser, Sov.Phys.JETP 46641 (1977).

2. J. Breitweg et. al., Eur. Phys. J. 67, 609 (1999) and references therein.

3. L. V. Gribov, E. M. Levin and M. G. Ryskin, Phys. Rep. 1001 (1983).

4. J. D. Bjorken, Phys. Rev. D27, 140 (1983).

5. L. McLerran and R. Venugopalan, Phys. Rev. D49 2233 (1994); D49 3352 (1994). 
6. Y. Kovchegov, Phys. Rev. D54, 5463 (1996); D55, 5445 (1997).

7. J. Jalilian-Marian, A. Kovner, L. McLerran and H. Weigert, Phys. Rev. D55 5414 (1997);

8. J. Jalilian-Marian, A. Kovner, A. Leonidov and H. Weigert Nucl. Phys. B504 415 (1997); Phys. Rev. D59 014014 (1999); J. Jalilian-Marian, A. Kovner and H. Weigert, Phys. Rev. D59 014015 (1999).

9. E. Iancu, A. Leonidov and L. McLerran, work in progress.

10. R. Venugopalan nucl-th/9808023.

11. M. Gyulassy and L. McLerran, Phys. Rev. C56 2219 (1997).

12. Al Mueller Nucl. Phys. B307, 34 (1988); B355, 115 (1990).

13. L. Mclerran and R. Venugopalan, Phys. Rev. D59 094002 (1999).

14. A. Kovner, L. McLerran, and H. Weigert Phys. Rev. D52, 3809 (1995); 6231 (1995).

15. A. Krasnitz and R. Venugopalan hep-ph/9808332, hep-ph/9809433.

16. S. Bass, B. Muller and W. Poschl, nucl-th/9808011.

17. A. Hebecker, Nucl. Phys. B505, 349 (1997).

18. W. Buchmuller, T. Gehrmann and A. Hebecker, Nucl. Phys. B537, 477 (1999).

19. L. Mclerran and Y. Kovchegov hep-ph/9903246 